\title{
UCID- $18574-81-4$
}

\section{MASTER}

H-DIVISION QUARTERLY REPORT

October through December 1981

March 5, 1982

This is an informal report intended primarily for internal or limited external distribution. The opinions and conclusions stated are those of the author and may or may not be those of the Laboratory.

Work performed under the auspices of the U.S. Department of Energy by the Lawrence I.ivermore Laboratory under ( ontract W-7405-kng-48. 


\section{DISCLAIMER}

This report was prepared as an account of work sponsored by an agency of the United States Government. Neither the United States Government nor any agency Thereof, nor any of their employees, makes any warranty, express or implied, or assumes any legal liability or responsibility for the accuracy, completeness, or usefulness of any information, apparatus, product, or process disclosed, or represents that its use would not infringe privately owned rights. Reference herein to any specific commercial product, process, or service by trade name, trademark, manufacturer, or otherwise does not necessarily constitute or imply its endorsement, recommendation, or favoring by the United States Government or any agency thereof. The views and opinions of authors expressed herein do not necessarily state or reflect those of the United States Government or any agency thereof. 


\section{DISCLAIMER}

Portions of this document may be illegible in electronic image products. Images are produced from the best available original document. 


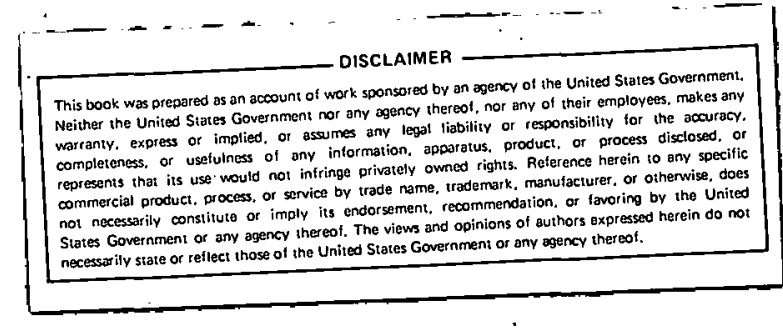

CONTENTS
UCID- $-18574-81-4$

DE82 009392

Theoretical physics

High pressure Structural Phase Transition in $\mathrm{Na}, \mathrm{Mg}$ and $\mathrm{Al}$

(J. A. Moriarty and A. K. MCMahan). . . . . . . . . . . . . . 1

Thermal and Compressibility Properties of the Alkali Metals

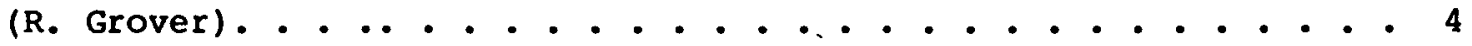

Electrical Conductivity in Hydrogen Plasmas (D. Boercker) . . . . 7

Plasma Pseudopotentials (F. J. Rogers, D. B. Boercker, and

H. E. DeWitt) . . . . . . . . . . . . . . . . . . . . . . 11

Theory of Thermonuclear Reaction Rates in strongly Coupled

Plasmas (H. E. DeWitt). . . . . . . . . . . . . . . . . . 14

Applications Phỵsics

Thermodynamic Consistency in BQL Interpolants (D. Daniel) • • . 20

Continuum Mechanics

Nonequilibrium Molecular Dynamics of Plastic Flow (W. G. Hoover). $\cdot 22$

Constitutive Models in the LLNL Hydrodynamic Codes

(W. Moss and L. Glenn). . . . . . . . . . . . . . . . . . . 24

Turbulence Interaction with Thermochemically lnert solid Pailicles

(A. C. Buckingham, W. J. Siekhaus, S. J. French, J. O. Keller,

J. Ellzey, G. Hubbard, and J. W. Daily) . . . . . . . . . . . 27

Experimental Physics

The Equation of State of Molecular Hydrogen and Deuterium from

Shock-Wave Experiments at $760 \mathrm{kbar}$ (W. J. Nellis, M. Ross,

A. C. Mitchell, M. van Thiel, and D. A. Young). . . . . . . . . 31

Nuclear-Explosive-Driven Equation-of-State Experiment

(W. J. Nellis, A. C. Mitchell, R. Olness, M. van Thiel) . . . . 35

DISTRIBUTAU OF THS DCOUMENT IS UHLIAIFED 
HIGH PRESSURE STRUCTURAL PHASE TRANSITION IN Na, Mg AND Al John A. Moriarty and A. K. McMahan

- We predict new, as yet undetected, structural phase transitions in $\mathrm{Na}, \mathrm{Mg}$ and $\mathrm{Al}$ under compression. These transitions arise from the effect of the downward motion of the initially empty 3d band. While this investigation was carried out up to ten-fold compression, a number of these transitions occur in the Mbar region and below, and should be observable by existing experimental techniques.

The ability to calculate the extremely small energy differences between different structures of a material, by first principles electron band theory, appears to be now within grasp. Successful comparisons between predictions of the linerar-muffin-tin-orbital (LMTO) method and the ab initio pseudopotential method used at Berkeley have already been reported for the case of $\mathrm{si}^{1}$ Here we report a much more extensive comparison between the LMTO method and the generalized pseudopotential theory ${ }^{2}$ (GPT) of the present first author for the case of $\mathrm{Mg}$ and $\mathrm{Al}$ up to ten-fold compression. Not only is there excellent qualitative agreement, but the major cause of quantitative differences is understood, and can be remedied. Furthermore, these successful comparisons serve to verify the atom-atom pair potentials generated by the GPT method in the present cases, suggesting that further calculations of the phonon spectra and lattice Grüneisen parameters from these potentials will be reliable.

The present calculations also predict a series of phase transitions in compressed $\mathrm{Na}, \mathrm{Mg}$ and $\mathrm{Al}$, of considerable interest in their own right. Figs. 1 and 2 show the results of total energy calculations for the bcc, hcp and fcc phases of these three materials as a function of relative volume, $\Omega / \Omega_{0}$. The total energies in each case are given relative to that for the fcc structure. Figs. $l(a)$ and $l(b)$ show our GPT results for Na and Mg, respectively, while Figs. $2(a)$ and $2(b)$ give a direct comparison of our LMTO and GPT results for $\mathrm{Al}$. As can be seen in Fig. 2, the qualitative agreement between GPT and LMTO predictions about phase stability in Al is excellent: both show large regions of fcc stability near normal volume and bcc stability at high compression separated by a small island of hcp stability. The 
qualitative agreement for Mg is comparable. No LMTO calculations on Na were attempted due to the tiny energy differences (typically $<0.1 \mathrm{mRy}$ ) in that metal.

On the basis of these calculations, we suggest the possible sequence of structures hcp $\rightarrow$ bcc $\rightarrow$ hcp in $\mathrm{Na}, \mathrm{hcp} \rightarrow \mathrm{bcc} \rightarrow \mathrm{fcc}$ in $\mathrm{Mg}$, and fcc $\rightarrow$ hcp $\rightarrow$ bcc in Al under increasing pressure. We estimate the initial hcp $\rightarrow$ bcc transitions in $\mathrm{Na}$ and $\mathrm{Mg}$, and $\mathrm{fCC} \rightarrow$ hcp transition in $\mathrm{Al}$, to occur at pressures of about 10, 570 and 1300 kbar at zero temperature, corresponding to $\Omega / \Omega_{0}=0.86,0.56$ and 0.58 respectively. These numbers reflect the addition of small zero-point energy contributions to the static lattice energy differences shown in the figures.

The structural energy differences in $\mathrm{Mg}$ and $\mathrm{Al}$ are profoundly influenced at small volumes by the presence of $d$ electrons. Under compression, the initially empty $3 d$ band moves downward toward the Fermi level $E_{F}$, narrowing relative to the $\mathrm{sp}$ bands in the process. In $\mathrm{Al}$, the LMTO $\mathrm{x}_{3}$ level (approximately the bottom of the $3 d$ band) moves below $E_{F}$ at $\Omega / \Omega_{0}=0.15$, at which point each wigner-Seitz cell contains about one d electron. We have directly assessed the effect of this d charge by repeating the LMTO calculations with d components removed from the angular momentum basis. The effect is striking, as shown in Fig. 3 for Al. The resultant bcc-fcc and hcp-fcc total energy differences now remain positive throughout the whole volume range considered. Analogous behavior is found for Mg. Thus without d electrons Mg_ and Al would remain hcp and fcc, respectively, down to at least $\Omega / \Omega_{0}=0.1$. Moreover, the addition of $f$ components to the basis changes the results in Figs. $2(a)$ and 3 only slightly.

The increasing quantitative discrepancies between GPT and LMTO results with decreasing volume, as seen in F1g. 3 , are probably due to differing treatments of d states. While the LMTO method is presumably treating the $d$ states accurately regardless of their spatial character (i.e., either nearly-free-electron or tight-binding like), the GPT, as applicd here in the simple-metal limit, is treating the d states accurately only to the extent that they remain nearly-free-electron like. Indeed, the purely p-like $x_{4}$, and $L_{2}$, levels are calculated to within a few percent of each other by the 
two techniques at all volumes. The sd hybrid $x_{1}$ level, on the other hand, shows close agreement only near normal volume, with the LMTO value moving progressively lower than the GPT value upon compression. This lowering is exactly the type of hybridization effect one expects from a narrowed d band centered above $E_{F}$ and suggests that the $3 d$ band in $A l$ is slowly developing some tight-binding-like character under pressure, which the present simple-metal form of GPT is not picking up. The natural remedy to this situation is to treat $A l$ as an empty-d-band metal in the manner of the heavy alkali and alkaline-earth metals. This possibility is currently under study. 
- Static and dynamic compression data have been analyzed together to obtain an improved representation of alkali metal compression properties. By combining both types of data, one obtains information on both the thermal and compressibility equation-of-state of the alkali metals over a large range of temperatures and densities which is of interest to nuclear reactor technology. This work is being prepared for the Handbook on Thermodynamic and Transport Properties of the Alkali Metals (IUPAC, edited by R. W. Ohse).

\section{Introduction}

Dynamic shock compression data extends the range of available experimental data on the alkali metals from compressions $n\left(\equiv \rho / \rho_{0}\right) \simeq 1.4$ to $\sim 3$ and up to temperatures ' $230 \mathrm{~T}_{M}$ (the melting temperature). Model equations-ofstate must be used to interpret this data since by itself the data is neither sufficiently accurate nor extensive to completely characterize the EOS. The model EOS must include the liquid phase since the shock compression data is almost completely in the liquid phase.

Although a simpler form of corresponding states theory can be used for alkali metal EOS at normal and lower densities, ${ }^{3}$ it is known that at higher densities, systematic variations with atomic number will occur due to influence of electronic core and $d$-band states. The phenomenological model EOS which we have used is based on three assumptions which appear reasonable in these circumstances:

1) The effective ion-ion forces are the same in both the solid and liquid metallic phases, relating the liquid EOS to a solid one, at the same $\rho$ and $\bar{T}$, in a simple way.

2) The changes in the thermal ion-ion forces (and therefore in Grüneisen cocfficient $\gamma_{G}$ ) are directly related to the changes in the compressibility of the metal which are caused by electron band anomalies.

3) Electron excitations make relatively small contributions to the "thermal" EOS of the alkali metals which can be represented hy a quasi-freeelectron approximation since all electron bands should be relatively wide. 
Assumption (2) is conventionally used in the form of a modified slater equation for $\gamma_{G}$, to reduce the shock compression data on metals. In addition it has been successfully applied, together with detailed band theory calculations, to the metals $\mathrm{Cs}$ and La where d-band crossings occur. ${ }^{4}$

\section{Method and Results}

Previous work on alkali metal compression data consisted for the most part in separate analyses of the static and dynamic data. ${ }^{5}$ In this work we have extended careful fits to normal compression data into the density range of shock data in order to obtain more reliable estimates of the EOS systematics of the alkali metals at high density.

In more detail, the present study. differs from previous work in two ways. First, the zero-degree isotherm pressure, which is used in a Grüneisen EOS model, is represented by an extended Birch equation

$$
\begin{aligned}
& P(n)=\frac{3}{2} B_{0} \eta^{5 / 3} \times \frac{\left[1+\frac{3}{4}\left(B_{0}^{\prime}-4\right) x+s_{2} x^{s_{3}}\right]}{1+s_{4} x^{S_{5}}} \\
& x \equiv n^{2 / 3}-1
\end{aligned}
$$

in terms of the normal density $\rho_{0}$, bulk modulus $B_{0}$ and its pressure derivative $B_{0}^{\prime}$. The additional coefficients $S_{2}$ and $S_{4}$ are used to make additions or subtractions to the first two, conventional terms and the exponents $s_{3}$ and $s_{3}$ to adjust the compression ranges of these modifications. The conventional Birch expansion is known to be the best systematic expansion for fitting normal compression data up to $\eta \simeq 1.4$. Secondly, the Grüneisen coefficient $\gamma_{G}$ in the thermal EOS is related to the zero degree isotherm, Eq. (1), by a generalized slater equation

$$
\gamma_{G}=v\left(\frac{\partial P}{\partial E^{\prime}} v_{, \text {sOIid }}=m-\frac{1}{6}+\frac{1}{2}\left(B^{\prime}-2 n / 3\right) /\left(1-\frac{2 n P}{3 B}\right)\right.
$$

In addition to the usual $n$ parameter, which selects the theories of slater, free volume, etc., Eq. (2) contains the parameter $m$ to allow $\gamma_{G}$ and its derivative $\varepsilon \equiv d \ln \gamma_{G} / d \ln v$ at normal density to be simply fitted to 
experimental data where available. Equation (2), retains the strong dependence of $\gamma_{G}$ on $\left.B^{\prime} \equiv \mathrm{dB} / \mathrm{dP}\right)_{\mathrm{T}=0}$ which is indicated by both theory and experimental data. Otherwise the liquid EOS model, the Lindemann law and the free electron excitation model are the same as in previous work. 5

Finally these models are accurately normalized to the experimental shock data $^{6}$ by adjusting the parameters remaining in Eqs. (1) and (2) after fitting the normal compression data, $\mathrm{B}_{0}, \mathrm{~B}_{0}^{\prime}$ and $\varepsilon$.

The results for $\mathrm{Li}, \mathrm{Na}, \mathrm{K}$, and $\mathrm{Rb}$ are summarized in the figures where both $\gamma_{G}$ and $\operatorname{lnB}$ (on the zero degree isotherm) are plotted against relative volume. Bulk modulus plots provide a sensitive method of comparing different types of experimental compression data with each other and our EOS model representation. It can be seen in Fig. 4 that normal density compressibility data is quite consistent and that for the worst case, Na, our model still represents the experimental data within the range of two recent sets of static data up to $30 \%$ compression. 3,7

In addition the $\ln B$ and $\gamma_{\mathrm{G}}$ plots show with increasing sensitivity the systematic variation of the high pressure EOS with atomic number. The high pressure stiffening of the zero degree isotherm seen in the lnB plot, Fig. 1, becomes stronger through sequence $\mathrm{Li} \rightarrow \mathrm{K}$, as is expected from the lowering of the electron d-bands and the larger cores of closed shell electrons. In the $\gamma\left(V / V_{0}\right)$ plot, Fig. 5, there is a rapid increase in the amount of lowering between $\mathrm{Na}$ and the heavier metals which may reflect the importance of d-band hybridization in the valence band. Moreover the rapid increase in $\gamma$ for $\mathrm{Rb}$ at the highest compressions may be an indication of the long expected electronic phase transition corresponding to the well known, $42 \mathrm{~kb}$ transition in Cs. Unfortunately the available shock data for $\mathrm{Rb}$ does not extend to the high pressure achieved in the other alkali metals. The inflection point in the $\gamma\left(\mathrm{V} / \mathrm{V}_{0}\right)$ for $\mathrm{Li}$ may be an artifact of the experimental data or the fitting procedure. In this connection it should also be noted that the Li EUS is based on a fit to shock data from two laboratories which are unusually inconsistent. Current experimental shock work on Li at this laboratory is being carried out to resolve this discrepancy. 
- The electrical conductivity of hydrogen plasmas is calculated using a recent result which relates the conductivity to the equilibrium structure of the plasma. A simple, analytic expression for the conductivity, designed to be accurate in the weak coupling limit gives good agreement with experimental results. A more elaborate calculation in more strongly coupled plasmas gives generally good agreement with molecular dynamics simulations at high density.

The electrical conductivity, $\sigma$, of a plasma can be related to the electron-ion collision frequency, $v$, through, 9

$$
\sigma=\lambda \frac{n e^{2}}{m v}
$$

where $n, m$, and $e$ are the electron number density, mass, and charge, respectively. The effects of electron-electron collisions are contained in the factor, $\lambda$. For weakly coupled plasmas, $\lambda$ is well-approximated by 1.93. 10 However, for strongly-coupled plasmas, $\lambda$ can be significantly less than this value. 11

The evaluation of the collision frequency has been discussed in detail elsewhere, $l$ and the principal result is that if $\lambda$ is selected to be 1.93 , and the conductivity is scaled to the electron plasma frequency, $\omega_{\mathrm{p}}=$ $\left(4 \pi n e^{2} / m\right)^{1 / 2}$, one obtains

$$
\sigma^{*} \equiv \frac{\sigma}{\omega_{p}}=\frac{1.93(3 \pi / 2)^{1 / 2}}{4 \pi \Gamma^{3 / 2} \Lambda}=\frac{0.3334}{\Gamma^{3 / 2} \Lambda}
$$

where $\Gamma=\operatorname{Be}^{2}(4 \pi n / 3)^{1 / 3}$ is the plasma coupling parameter, and $\Lambda$ is a generalization of the Coulomb logarithm defined by,

$$
\Lambda \equiv \frac{1}{4 \pi B e^{2}} \int_{0}^{\infty} d k k e^{-B h^{2} k^{2} / 8 m} \frac{c_{e j}(k)}{\left(\varepsilon_{e}(k)\right)^{2}} s_{i i}(k)
$$

In the above, $\beta$ is the inverse temperature and $h$ is Planck's constant over $2 \pi$. The functions $s_{i j}(k)$ and $c_{e i}(k)$ are the ion-ion structure factor 
and the electron-ion direct correlation function, respectively. The screening factor, $\varepsilon_{e^{\prime}}$ is related to the electron-electron direct correlation function through,

$$
\varepsilon_{e}(k)=1-n c_{e e}(k)
$$

In the Debye-Hückel limit the integral in $\mathrm{Eg}$. (3) may be done analytically with the result

$$
\begin{aligned}
\Lambda_{D H} & =e^{1 / \alpha} E_{1}\left(\frac{1}{\alpha}\right)-\frac{1}{2} e^{1 / 2 \alpha} E_{1}\left(\frac{1}{2 \alpha}\right) \\
& \approx \frac{1}{2}(\ln \alpha-1.27)
\end{aligned}
$$

in which $\alpha=\left(\mathrm{m} / \pi \beta^{2} \mathrm{~h}^{2} \mathrm{ne}^{2}\right)$ and $\mathrm{E}_{I}$ is the exponential integral. ${ }^{12}$ The second line in Eq. (5) applies when $\alpha \gg$ 1. Table I shows experimentally measured conductivities $^{13}$ in hydrogen and compares them to the results calculated from Eqs. (2) and (5) and from the Spitzer theory. ${ }^{14}$ In general, the present theory agrees with the experiment to within about 158 and is somewhat better than the spitzer theory.

of course, this would not be of much significance if it were not for the fact that Eq. (3), as well as Eq. (5), can be used in regions where spitzer fails. In Table II the conductivity found from molecular dynamics simulation of a hydrogen plasma ${ }^{15}$ is compared to calculations using Eq. (2) with both Eq. (5) and the direct numerical evaluation of Eq. (3). The rather large disagreement at the largest $\Gamma$ value can be attributed to two sources. First is the failure of the approximation, $\lambda=1.93$, and second is the difference in the way quantum mechanics is treated in this theory and in the molecular dynamics. Work directed at correcting the former shortcoming is progressing rapidly.

In summary, an expression for the electrical conductivity has been presented which can be applied to weak and moderately coupled plasmas $(\Gamma \leq 2)$. In the weak coupling limit, it agrees with available experimental data to within about $15 \%$ and is an improvement over the standard spitzer expression. For stronger coupling it is in general agreement with molecular dynamics 
simulations. It is hoped that in the near future the more elaborate pseudopotentials of Rogers ${ }^{16}$ can be used to study the conductivity of more complicated and interesting plasmas. 
Table I. Comparison of theoretical and experimental values of $\sigma^{*}$.

\begin{tabular}{ccccc}
\hline$T\left({ }^{\circ} \mathrm{K}\right)$ & $\Gamma$ & $\sigma^{*}$ & $\sigma_{\text {Spitzer }}^{*}$ & $\sigma_{\text {exp }}^{*}$ \\
\hline 15,400 & 0.165 & 1.34 & 1.57 & 1.16 \\
18,750 & 0.161 & 1.41 & 1.61 & 1.32 \\
21,500 & 0.144 & 1.62 & 1.81 & 1.55 \\
\hline
\end{tabular}

Table II. Comparison with molecular dynamics results $\left(r_{s}=(4 \pi n / 3)^{-1 / 3 / a_{0}}\right.$ ' $a_{0}=$ Bohr radius).

\begin{tabular}{ccccc}
\hline$\Gamma$ & $\mathbf{r}_{\mathrm{S}}$ & $\sigma^{*}$ & $\sigma_{\mathrm{DH}}^{*}$ & $\sigma_{\mathrm{MD}}^{*}$ \\
\hline 0.5 & 0.4 & 4.00 & 3.55 & 3.6 \\
0.5 & 1.0 & 1.93 & 1.87 & 3.15 \\
2.0 & 1.0 & 2.39 & 3.11 & 1.1 \\
\hline
\end{tabular}


PLASMA PSEUDOPOTENTIALS

F. J. Rogers, D. B. Boercker, and H. E. DeWitt

- Numerical calculations of plasma pseudopotentials that preclude strong bound states have been carried out. Application to the hypernetted chain integral equation are discussed.

The thermodynamic properties of classical models of dense fluids and plasmas can be obtained from Monte Carlo, molecular dynamics, and integral equation methods. However, realistic models of electron-ion plasmas require a quantum-mechanical treatment. It is very difficult to apply the standard methods in this case. An approximate procedure for doing this is to introduce a pseudopotential that has quantum effects built-in at short-distances, but which goes over to the Coulomb potential at distances large compared to the deBroglie wavelength. This removes the short-ranged divergence in the electron-ion distribution function, $g_{e i}$ ' which is present in the classical problem. At low temperature, due to the formation of composite particles, numerical difficulties still exist.

The low temperature numerical difficulties can be circumvented by separating the slater sum into a bound state part, due to low lying bound states, and an interaction part due to continuum states and high lying bound states. Larkin ${ }^{17}$ has shown the proper way to carry out this separation in the partition function. Essentially the partition function is separated into a convergent part and a non-convergent part. The resulting convergent bound state part is referred to as the Planck-Larkin partition function.

The corresponding separation in the slater sum is

$$
s_{e i}=s_{e i}^{B S}+s_{e i}^{I}
$$

where

$$
\mathrm{s}_{\mathrm{ei}}^{\mathrm{BS}}=\left(\frac{2 \pi \beta h^{2}}{\mu_{\mathrm{e} i}}\right)^{3 / 2} \sum_{n \ell}(2 \ell+1) \psi_{n \ell}^{*}(r)\left(e^{-\beta E n \ell}-1+\beta E_{n \ell}\right) \psi_{n \ell}(r)
$$




$$
\begin{aligned}
\mathrm{s}_{\mathrm{e} i}^{\mathrm{I}} & =\left(\frac{2 \pi \beta \mathrm{h}^{2}}{\mu_{\mathrm{e} i}}\right)^{3 / 2} \sum_{\ell}(2 \ell+1) \int_{0}^{\infty} \psi_{\ell}^{*}(\mathrm{r}, \mathrm{p}) \mathrm{e}^{-\beta \mathrm{p}^{2} / \mu} \mathrm{e} \alpha \psi_{\ell}(\mathrm{r}, \mathrm{p}) \mathrm{dp} \\
& -\sum_{n \ell}(2 \ell+1) \psi_{n \ell}^{*}(\mathrm{r})\left(1-\beta E_{n \ell}\right) \psi_{n \ell}(\mathrm{r})
\end{aligned}
$$

where the symbols have their usual meaning. Barker ${ }^{18}$ obtained pseudopotentials according to

$$
B_{\text {ei }}(r, B)=-\ln s_{e i}
$$

In the present work we will define a pseudopotential which precludes strong bound states according to

$$
B u_{e i}(r, \beta)=-\ln s_{p i}^{I}
$$

We will refer to this as the Planck-Larkin pseudopotential.

The wavefunctions required to evaluate $\mathrm{Eq}$. (1) were obtained numerically. Previous work on this problem has been analytic and limited to hydrogen. The interest here is more general. We are interested ultimately in obtaining the equation of state and transport properties of dense partially ionized plasmas. We will treat composite particles by utilizing the electron-ion effective potentials, $v_{\text {el }}$ presented in Ref. 19.

Numerical calculations are difficult for a potential having a coulomb tail. This presents no particular difficulty since guantum effects are only important for $r<\lambda_{i j}$, the deBroglie wavelength. The procedure is to carry out the calculations for screened potentials having a tail of the form $\left(^{-r / \alpha_{i j}}\right) / r$ where $\alpha_{i j} \gg \lambda_{i j}$. Small deviations from the isolated atom limit $\left(\alpha_{i j}+\infty\right)$ are easily removed by extrapolation. In the current work hydrogen is used to illustrate the method.

Figure 6 compares the Barker and Planck-Larkin electron-proton pseudopotentials for $k T=2 \mathrm{eV}, \rho=0.0166 \mathrm{~g} / \mathrm{cm}^{3}$ and $\alpha_{\text {ep }}=80 \alpha_{0}$. The 
pseudopotential given by Deutsch ${ }^{20}$ and used by Hansen and McDonald ${ }^{21}$ in molecular dynamics simulations of an electron-proton plasma is also shown in Fig. 6. For a potential with a screened tail it has the form

$$
u_{i j}=\frac{z_{i}{ }{ }_{j} e^{2}}{r}\left(e^{-r / \alpha_{i j}}-e^{-c r}\right),
$$

where $c=1 / \lambda_{i j}$. The constant $c$ was chosen to match $g_{i j}(0)$ for $k T>I$ Ryd. The Deutsch pseudopotential is only approximate and is used in applications because of its analytic simplicity.

At high temperature ( $k T>1$ Ryd) the Deutsch pseudopotential is rather close to the Planck-Larkin pseudopotential. As the temperature is reduced below a Rydberg due to partial inclusion of strong bound states, the Deutsch pseudopotential moves lower than the Planck-Larkin pseudopotential (see Figs. 6 and 7). This has important implications in some hypernetted chain calculations with the Deutsch pseudopotential for a three component $e-p-\mathrm{Fe}^{24+}$ plasma we have been studying. In this work it was noted that the large peaks in $\mathrm{eFe}^{24+}$ near $\mathrm{r}=0$ caused the integral equation iteration procedure to become unstable. Since the Planck-Larkin pseudopotential is weaker it gives electron-ion distribution functions which are less strongly peaked. This should aid in stabilizing the hypernetted-chain iteration procedure.

Figure 8 compares the electron-proton hypernetted chain distribution functions given by the Deutsch and Planck-Larkin pseudopotentials for $k T=$ $2 \mathrm{eV}$ and $\alpha_{e p}=\infty$. Since the electron-electron and proton-proton interactions do not produce bound states the Deutsch pseudopotential was used for these interactions. Hypernetted chain calculations for both pseudopotentials are stable in this example, but $g_{e p}(0)$ for the Deutsch pseudopotential is 2 $1 / 2$ times larger than the Planck-Larkin pseudopotential. At lower temperatures and/or lower densities the hypernetted chain calculations for the Deutsch pseudopotential become unstable, while calculations with the PlanckLarkin pseudopotential are stable. 
THEORY OF THERMONUCLEAR REACTION RATES IN STRONGLY COUPLED PLASMAS Hugh E. Dewitt

- The Dewitt, Graboske, Cooper theory of enhancement of thermonuclear reaction rates in stellar interiors is modified by use of Monte Carlo results for strongly coupled plasma mixtures. A mixing formula proposed by Rosenfeld is shown to be valid in the weak and strong coupling limits and leads to a reaction rate enhancement valid for most LLNL applications. The result may be used for calculating the reaction rate of DT in the presence of.high $\mathrm{Z}$ impurities.

The thermonuclear reaction rate of charged particles in high temperature dense plasmas is enhanced by a lowering of the loulomb barrier due tu correlational effects of the plasma in the vicinity of a reacting pair of charges. A detailed theory of this enhancement effect was given by Dewitt, Graboske, and Cooper (DGC) in 1973 for intermediate and strong coupling. 22 The DGC formulae were largely based on the Monte Carlo study of the one component plasma (OCP) of Brush, Sahlin, and Teller, ${ }^{23}$ and consequently were most dependable for reacting pairs of charges in a plasma of one ionic charge, for example, DT reactions in laser fusion experiments. The theory of 1973 was not accurate enough to handle reactions of pairs with very different charges nor situations involving DT reactions in the presence of high $\mathrm{z}$ impurities. Due to recent theoretical and numerical simulation work on strongly coupled plasma mixtures, it is now possible to markedly improve the DGC theory of the screening enhancement of the reaction rate.

We consider a plasma of several different ionic species with charge number, $z_{1}, z_{2}, z_{v}$, and with chemical compositions, $x_{i}=N_{i} / N(N=$ $\left.\mathrm{N}_{1}+\mathrm{N}_{2}+\ldots+\mathrm{N}_{v}\right)$. The reacting pair, $\left(\mathrm{z}_{1}, \mathrm{z}_{2}\right)$ results in a composite particle of charge $\left(z_{1}+z_{2}\right)$. The reacting charges are part of a thermal distribution, $B=1 / k T$, and can react if they surmount the repulsive potential barrier defined by the potential of mean force, $w_{12}(r)$, and obtained from the equilibrium pair distribution:

$$
g_{12}(r)=\exp \left(-B w_{12}(r)\right)=\exp \left\{-B z_{1} z_{2} e^{2} / r+H_{12}(r)\right\}
$$


$\mathrm{H}_{12}(\mathrm{r})$ is the screening function that reduces the repulsive Coulomb potential, $z_{1} z_{2} e^{2} / r$, and at a zero particle separation gives the reaction rate enhancement as:

$$
\left.\langle\sigma v\rangle_{12, \text { plasma }} \cong \mathrm{e}^{-\mathrm{H}_{12}(0)}<\sigma v\right\rangle_{12} \text {, vacuum }
$$

The complete calculation of the reaction rate is also influenced by the $r$ dependence of $\mathrm{H}_{12}(r)$ as the charges move into the potential barrier 24,25 but here only the lowering of the barrier by $\mathrm{H}_{12}(0)$ will be discussed. The Coulombic effects that enhance the reaction rate are dominated in intermediate and strong coupling by the interactions among the various ions; the electrons at sufficiently high density form a degenerate neutralizing background which, for the present discussion, will be considered as a rigid background. Realistic electron screening can be incorporated at a later stage. With the assumption of classical ions moving in a rigid neutralizing background the interaction Helmholtz free energy may be written as:

$$
B F / N=f_{v}\left(\Gamma_{o} \bar{z}^{1 / 3}, x_{1}, z_{1}, x_{2}, z_{2}, \ldots x_{v^{\prime}} z_{v}\right)
$$

with

$$
\begin{aligned}
& \Gamma_{0}=\beta e^{2} / a, a=\left(\frac{4 \pi}{3} \frac{N}{v}\right)^{-1 / 3} \\
& z=x_{1} z_{1}+x_{2} z_{2}+\cdots \cdot+x_{v} z_{v}
\end{aligned}
$$

The interaction internal energy as obtained from the Helmholtz free energy' is:

$$
\beta U / N=\beta \frac{\partial(\beta \mathbf{F} / N)}{\partial \Gamma}=\Gamma_{0} \frac{\partial \mathbf{f}}{\partial \Gamma}
$$

In practice the definitive data for the internal energy are obtained by Monte Carlo simulation of the classical plasma mixtures and the free energy function is constructed by integrating Eq. 4 . 
DGC showed that the Coulomb barrier lowering, $\mathrm{H}_{12}(0)$, was also a thermodynamic function, namely the difference of the free energies before and after the reaction, $z_{1}+z_{2}+\left(z_{1}+z_{2}\right):^{1}$

$$
\begin{aligned}
& \mathrm{H}_{12}(0)=-(N-1) f_{v+l}\left(\Gamma_{0} \bar{z}^{1 / 3}, \frac{N_{1}-1}{N-I}, z_{1}, \frac{N_{2}-1}{N-I}, z_{2}, \cdots, \frac{N_{v}}{N-1},\right. \\
& \left.\frac{1}{N-1},\left(z_{1}+z_{2}\right)\right)+N_{v}\left(\Gamma_{0} \bar{z}^{-1 / 3}, \frac{N_{1}}{N}, z_{1}, \frac{N_{2}}{N}, z_{2}, \cdots \cdot \frac{N_{v}}{N}, z_{v}\right)
\end{aligned}
$$

In 1973 this relation provided the structure of the DGC theory but because of lack of information about strongly coupled plasma mixtures it coula not be used directly. Instead DGC had to construct the $\mathrm{H}_{12}(0)$ published at that time by using the limited OCP data for $g(r)$ and extrapolating $H(r)$ to $r=0$. Now with an abundance of very accurate $O C P$ and binary ionic mixture Monte Carlo data it is possible to use Eq. 5 directly. The calculation requires two things:

i) exact OCP energy data from which the OCP Helmholtz free energy funcion, $f_{l}(\Gamma)$, can be constructed.

ii) an accurate mixture rule from which $v$ component free energy $f_{v}$ can be obtained from $f_{I}\left(I^{\prime}\right)$.

The OCP energy results are known from solution of the hyper-netted chain equation (HNC) $(0<\Gamma<.8)$, and from long Monte Carlo simulations (collaboration with $W$. Slattery and G. Doolen at LANL) 26,27

The mixture problem is awkward because two limits must be satisfied. For weak coupling the Debye-Hückel theory gives an exact result:

$$
B F_{V} / N=f_{V}=-\frac{\left(\sqrt{ } 3 \Gamma_{0}^{3 / 2}\right)-\overline{2}^{3 / 2}}{3} z^{3}, \Gamma_{0} \ll 1
$$


while in strong coupling the mixture is dominated by the ion-sphere result:

$$
B F_{V} / \mathrm{N} \simeq-\frac{9}{10} \Gamma_{0} \bar{z}^{-1 / 3} \overline{z^{5 / 3}}, \Gamma_{0} \ll 1
$$

Hansen, Torrie, and Vieillefosse ${ }^{28}$ proposed a 'linear' mixture formula which satisfied their binary mixture solutions of coupled HNC equations and a small amount of binary mixture Monte carlo data:

$$
f_{v, \text { linear }}=\sum_{i=1}^{\cup} x_{i} f_{1}\left(\Gamma_{0} \bar{z}^{-1 / 3} z_{i}{ }^{5 / 3}\right)
$$

This empirical result was checked with more extensive mixture MC and HNC calculations at LLNL $^{29}$ and found to be in good agreement with the numerical data. The Hansen, et al. "linear" law clearly gives the expected ion-sphere result for strong coupling, but leads to an incorrect result for weak coupling. Consequently, efforts to calculate $\mathrm{H}_{12}(0)$ for intermediate and even strong coupling using the 'linear' law would be suspect. Recently Rosenfeld ${ }^{30}$ studied the mixture problem by using known exact lower bounds for Helmholtz free energy ${ }^{31}$ for weak and strong coupling. By minimizing the free energy with respect to both lower bounds he was able to derive an approximate, but highly accurate mixing rule:

$$
f_{V, R}=\left(\frac{\bar{z}}{z^{2}}\right) \sum_{i=1}^{\nu} x_{i} z_{i} f_{1}\left(\Gamma_{o} \bar{z}^{-1 / 3} z_{i} 2 / 3 \bar{z} / \bar{z}\right)
$$

This expression for the plasma free energy mixture recovers both known limits, Eqs. 6 and 7, and consequently it can be used for intermediate coupling. When applied to the LLNL Monte Carlo mixture data ${ }^{29}$ the agreement is slightly less good than the agreement with the linear formula, Eq. 8, but the difference is near the limit of the accuracy of the Monte carlo simulation.

In applying the Rosenfeld mixture formula to the evaluation of $\mathrm{H}_{12}(0)$ using Eq. 5 some care must be maintained to pick up contributions of order $1 / \mathrm{N}_{1}$ and $1 / \mathrm{N}$. The result is: 


$$
\begin{aligned}
& H_{12}(0)=-\left(\frac{\bar{z}}{z^{2}}\right)\left\{\left(z_{1}+z_{2}\right) f_{1}\left(\Gamma_{12}\right)-z_{1} f_{1}\left(\Gamma_{1}\right)-z_{2} f_{1}\left(\Gamma_{2}\right)\right\} \\
& +\frac{2 z_{1} z_{2} \bar{z}}{\left(z^{2}\right)^{2}} \quad \sum_{i=1}^{\cup} x_{i} z_{i}\left(f_{1}\left(\Gamma_{i}\right)-\Gamma_{i} \frac{d f_{1}}{d \Gamma_{i}}\right)
\end{aligned}
$$

where

$$
\Gamma_{i}=\Gamma_{o} \bar{z}^{1 / 3} z_{i}^{2 / 3} z^{-\overline{2}} / \bar{z}
$$

The derivative term in this expression is essential to give the weak sounling limit:

$$
\mathrm{H}_{12}(0)=-\mathrm{z}_{1} \mathrm{z}_{2} \mathrm{z}^{-\mathrm{z}^{1 / 2}}\left(\sqrt{3} \Gamma_{0}^{3 / 2}\right)
$$

The OCP free energy function vs. $\Gamma$ is shown in Fig. 9. For strong coupling the OCP free energy function has the form:

$$
f_{1}(\Gamma)=-A \Gamma+4 B \Gamma^{1 / 4}-C \ln \Gamma-D-4 E / \Gamma^{1 / 4} \cdot \cdots
$$

for $\Gamma>.8$. Note that the Madelung term of the strong coupling free energy, - $\mathrm{Al}^{\prime}$, in $\mathrm{Eq}$. 11 gives the expected ion-sphere result:

$$
\mathrm{H}_{12}(0) \cong \mathrm{A} \Gamma_{0} \overline{\mathrm{z}}^{-1 / 3}\left\{\left(\mathrm{z}_{1}+\mathrm{z}_{2}\right)^{5 / 3}-\mathrm{z}_{1}^{5 / 3}-\mathrm{z}_{2}^{5 / 3}\right\}
$$

with $A=.8979$

Equation 10 is the desired expression for the lowering of the Coulomb barrier, and should be used in place of Eq. 53 in the 1973 paper of DGC. Precise limits on the validity of these results have not been established yet, but it is expected that they will be adequate for nuclear reaction rate calculations for DT reactions in the presence of one or more high $z$ impurities. 
Future work on the reacion rate problem will focus on proper incorporation of the electrons allowing for polarization of the electrons by linear response theory, and a direct calculation of $\mathrm{H}_{12}$ from coupled HNC equations modified to include bridge graph corrections. 
THERMODYNAMIC CONSISTENCY IN BQL INTERPOLANTS

Doug Daniel

- We examine the mathematical limitations to thermodynamic consistency imposed by BQL pressure and energy interpolants used for EOS representations. The answer impacts directly on how well an energytransport hydrocode can adhere to the first law of thermodynamics.

There is some concern within the user community about thermodynamic consistency of equations of state (EOS) represented by bi-quadratic-Laqranqe (BQL) interpolants. To solve the problem we applied the appropriate Maxwell relation to the interpolants and examined the resultant functional forms. We choose to derive the energy interpolant from a BQL pressure surface. We then compare the consistent energy functional with a BQL energy interpolant.

The interpolants used in BQL representations are:

$$
\begin{aligned}
& P(\rho, T)=\sum_{i=0}^{2} \sum_{j=0}^{2} b_{i j} \rho^{i_{T}} \\
& E(\cap, T)=\sum_{i=0}^{2} \sum_{j=0}^{2} a_{i j} \rho^{i_{T} j}
\end{aligned}
$$

The Maxwell relation with $\rho, T$ independent variable is easily derived from the first law of thermodynamics

$$
\mathrm{dE}=\operatorname{TdS}+\frac{P}{\rho^{2}} \mathrm{~d} \rho
$$

and the definition of Helmholtz free energy

$$
F=E-T S \text {. }
$$


Hence

$$
T\left(\frac{\partial P}{\partial T}\right)_{\rho}+\rho^{2}\left(\frac{\partial E}{\partial \rho}\right)_{T}=P
$$

is the Maxwell relation in $\rho, T$ space.

By integration, we have

$$
E-E_{0}(T)=\int\left[P-T\left(\frac{\partial P}{\partial T}\right) \rho_{\rho}\right] \frac{1}{\rho^{2}} d \rho
$$

We obtain the consistent energy interpolant by imposing the above integration on the $B Q L$ pressure function (1)

$$
E-E_{0}(T)=\sum_{j=0}^{2} T^{j}(1-j)\left[\frac{-b_{0 j}}{p}+b_{1 j} \ln \rho+b_{2 j} \rho\right]
$$

One can determine by comparing (2) and (3) that $B Q L$ pressure and energies cannot be rigorously consistent. Clearly, the degree of inconsistency is determined by the $\rho, T$ mesh and the order of approximation of (3) to (2). A Taylor's expansion of eq. (3) minus eq. (1) shows the approximation to be quite good in $\mathrm{T}$ but first order in $\rho$ only for small $\Delta \rho$ or large density.

One can conclude that for the best case (when the primary data base is thermodynamically consistent and the fit reproduces the data exactly) that a BQL energy surface can intersect a consistent energy surface only at the mesh points and be rigorously consistent at no more than nine points. 
CONTINUUM MECHANICS

NONEQUILIBRIUM MOLECULAR DYNAMICS OF PLASTIC FLOW

William G. Hoover

- Computer simulations of high strain-rate plastic flow, in twodimensional solids, reveal the dependence of energy and stress on density, strain-rate, temperature, and the scale of flow.

Nonequilibrium molecular dynamics is being developed to simulate constrained flow processes. This technique is now being applied to steady flows in two-dimensional periodic solids in order to relate high-strain-rate deformation to microscopic models of plasticity. The original nonequilibrium molecular dynamics work ${ }^{32}$ established that periodic rescaling of atomic momenta (relative to the local hydrodynamic velocity) could be used to impose constant velocity and constant temperature boundary conditions. These could then be used to simulate viscous heat conductiny fluws.

More recently a periodic scaling of space has been introduced directly into Hamilton's equations of motion. This technique can be used to maintain either a constant strain rate ${ }^{33}$ or a constant stress ${ }^{34}$ on a fluid or solid system. Our current work combines these two ideas. ${ }^{35}$ The adiabatic Hamiltonian equations of motion (two dimensions) for a particle undergoing steady shear, with $d u_{x} / d y=\varepsilon$ are

$$
\dot{x}=\left(F_{x} / m\right)+\dot{F}_{y} ; \dot{y}-\left(p_{y} / m\right), \dot{p}_{x}=F_{x} ; \dot{p}_{y}=F_{y}-\dot{F}_{x} .
$$

The velocity dependent force $-\dot{\varepsilon} p_{x}$ can be thought of as a coriolis force arising from the rotational nature of simple shear flow. If to these adiabatic equations of motion are added "frictional" contributions, chosen to maintain the kinetic temperature constant, $(d / d t) \Sigma p_{x}^{2}=(d / d t) \Sigma p_{y}^{2}=0$, then the friction constants $\zeta_{x}$ and $\zeta_{y}$ can be evaluated, as functions of time: 


$$
\begin{array}{lll}
\dot{\mathrm{p}}_{\mathrm{x}}=\mathrm{F}_{\mathrm{x}}-\zeta_{\mathrm{x}} \mathrm{p}_{\mathrm{x}} & ; & \zeta_{\mathrm{x}}=\Sigma \mathrm{p}_{\mathrm{x}} \mathrm{F}_{\mathrm{x}} / \Sigma \mathrm{p}_{\mathrm{x}}{ }^{2} \\
\dot{\mathrm{p}}_{\mathrm{y}}=\mathrm{F}_{\mathrm{y}}-\zeta_{\mathrm{y}} \mathrm{p}_{\mathrm{y}}-\dot{\mathrm{e}}_{\mathrm{x}} ; & \zeta_{\mathrm{y}}=\Sigma\left(\mathrm{p}_{\mathrm{y}} \mathrm{F}_{\mathrm{y}}-{\dot{\varepsilon p_{x}}}_{\mathrm{p}}\right) / \Sigma \mathrm{p}_{\mathrm{y}}^{2}
\end{array}
$$

These deforming isothermal equations have been implemented on the CRAY and 7600 computers. Tests with zero strain rate show that the equations of motion are stable and correctly reproduce the thermodynamic properties of quasiharmonic crystals. Tests with nonzero strain rates on periodic crystals containing a few hundred particles are also stable and allow us to measure the energy and the stress tensor as functions of thermodynamic state (including strain rate and system size). These simulations, when complete, will be related to ongoing theoretical studies $^{36}$ of the properties of edge dislocations in atomistic crystals. The latter work has established the core energy of these dislocations as well as the functional relationships between dislocation velocity and stress. 37 
CONSTITUTIVE MODELS IN THE LLNL HYDRODYNAMIC CODES

W. Moss and L. Glenn

- A program to review critically the constitutive models that are being used in our hydrodynamic codes was initiated in october 1981. We are examining the validity of the fundamental assumptions, self consistency, previously neglected physics, and uniqueness (unambiguous determination of parameters) of the models. The elastic-plastic constitutive model, which consists of a von Mises yield criterion, Prandtl-Reuss flow rule, and a pressure dependent yield strength is being studied currently. A pressure dependent yield otrongth, with a consistent (assnriated) flow rule causes plastic dilatation, which increases the pressure in the material. Concurrent with our clastio-plastic constitutive model development, we have modified an existing computer code (AFTON), so that any constitutive model we develop can be easily tested.

Our elastic-plastic constitutive model is based on the von Mises theory of the plastic potential. 38 A convex (closed) yield surface is postulated to exist:

$$
f=0
$$

For isotropic materials, f must be a function of stress invariants. Stress states inside the yield surface, $f<0$, represent elastic states, whereas, stress states on the yield surface, $f=0$ represeil plastic statea. strces states outside the yield surface, $f>0$, are not allowed. The flow rule obtained from plastic potential theory is:

$$
\operatorname{de}_{\mathbf{i j}}^{p}=\lambda \frac{\partial \mathrm{f}}{\partial \sigma_{\mathbf{i j}}} ; \lambda>0 \text {, }
$$

where $d_{i j} \underset{i j}{p}$ is the incremental plastic strain, $\sigma_{i j}$ is the stress, and $\lambda$ is a positive constant. Equation (2) is equivalent to requiring that plastic flow is normal to the yield surface. Equation (2) is called an associated flow rule, because the plastic strain increments are associated with the yield function. 
For our elastic-plastic constitutive model, we choose $f$ to be identical to the form used in the hydrodynamic code model, which is a von Mises yield criterion:

$$
f=J_{2} \cdot-\frac{1}{3} y^{2} ; J_{2}^{\prime}=\frac{1}{2} s_{i j} s_{i j}
$$

where $J_{2}{ }^{\prime}$ is the second invariant of the stress deviator tensor, and is a measure of the mean square shear stress. $Y$ is a function of pressure, temperature, and equivalent plastic strain.

We obtain flow rules from Eq. (2), by considering two cases: (i) $Y$ is pressure independent, and (ii) $Y$ is pressure dependent.

When $Y=Y(P), E q . \quad(2)$ yields

$$
\operatorname{de}_{i j}^{p}=\lambda s_{i j}
$$

Equation (4a) is the Prandtl-Reuss flow rule. We note that the incremental plastic dilatation, which is the trace of the incremental plastic strain tensor, is zero:

$$
\mathrm{de}_{i \mathrm{i}}^{\mathrm{p}}=\lambda \mathrm{s}_{\mathbf{i} \mathrm{i}}=0
$$

When $Y=Y(P), E g \cdot(2)$ yields

$$
\begin{aligned}
& \operatorname{de}_{i j}^{p}=\dot{\lambda}\left[s_{i j}-\frac{2}{3} Y \frac{\partial Y}{\partial \sigma_{i j}}\right] \\
& \operatorname{de}_{11}^{p}=-\frac{2}{3} \lambda Y \frac{\partial Y}{\partial U_{i i}}=\frac{2}{3} \lambda Y \frac{Y Y}{\partial F}
\end{aligned}
$$

Equation (5a) is the Prandtl-Reuss flow rule, with an additional term that is due to the stress dependence of $Y$. Equation (5b) shows that the plastic dilatation is nonzero, if the yield strength is a function of pressure. 
The hydrodynamic code model consists of Eqs. (1), (3), and (4a), Our model would be identical to the former, if the yield strength was pressure independent. However, Eq. (5) must replace Eq. (4), in order to have a self-consistent theory.

We note that the von Mises theory of the plastic potential is one of many elastic-plastic theories. More complicated yield functions can be postulated and nonassociated flow rules may be used. We are investigating the plastic potential theory because it is similar to our present code model, but adds the potentially important effects of plastic dilatation.

The physical significance of Eq. (5) is that plastic dilatation increases the pressure in the material. Figure 10 shows how this occurs. In the riyuie, $\checkmark J_{2}{ }^{\prime}$ is plotted, as a function of $P$. We assume that at time $n-1$, the material is elastic $(\mathrm{f}<0)$, therefore, the point $\left(\mathrm{P}_{n-1}, /\left(\mathrm{J}_{2}{ }^{\prime}\right)_{n-1}\right)$ is inside the yield surface $f=0$, which is a function of pressure. A trial compression produces a change in pressure $\mathrm{dP}^{\mathrm{T}}$. In some codes, the trial compression is calculated from Newton's Laws. If the resulting $V_{J}{ }_{2} T$ (calculated by assuming the trial compression is totally elastic), which corresponds to $\mathrm{P}^{\mathrm{T}}=\mathrm{P}_{\mathrm{n}-1}+\mathrm{dP}^{\mathrm{T}}$, is inside the yleld surface, then the stress state is elastic and the calculation is finished. However, if $\left(P^{T}\right.$, $\sqrt{J}_{2}, \mathrm{~T}$, corresponds to a virtual state outside the yield surface, then a flow rule is needed to determine the path back to the yield surface. The Prandtl-Reuss flow rule allows no plastic dilatation; lhelefore, the path to the yield surface occurs at constant pressure, $\mathrm{P}^{\mathrm{T}}$. Plastic potential theory requires that plastic flow is normal to the yield surface, therefore, the pressure increases by $\mathrm{dP}^{\mathrm{P}}$, as shown in the figure. The total change in pressure is $d P=d P^{T}+d P^{P}$. We note that the flow rule (5) can couple the elastic-plastic constitutive model to the equation of state, thus changing the effective bulk modulus of the material. Future work will consist of testing this elastic-plastic model by examining the effects of plastic dilatation on wave profiles. 
TURBULENCE INTERACTION WITH THERMOCHEMICALLY INERT SOLID PARTICLES

A. C. Buckingham, W. J. Siekhaus, ${ }^{\star}$ S. J. French,
J. O. Keller, ${ }^{+}$J. Ellzey, ${ }^{+}$, G. Hubbard, ${ }^{+}$J. W. Daily

- We are theoretically and experimentally investigating the interactions between turbulence and dispersed inert particles. We are interested in the apparent reduction in turbulent intensity associated with thermochemically inert solid particle dispersal in developing free shear layers. Two plane, incompressible, shear flows are studied. One forms over a rearward facing step and the other over a parallel splitter plate. Both evolve in a rectangular test section driven by a steady, mean, incompressible stream. Computations on these flow configurations are being prepared. The computations include Reynolds' averaged unsteady Navier-stokes gas field solutions combined with separate Langrangean particle field solutions. Random statistical interactions simulate the connection between the two fields. This study is a part of our research on fundamental mechanisms of hot gas erosion which is funded by the Department of the Army, U.S. Army Research Office.

It seems intuitively reasonable that, in a turbulent flow, particle inertial effects, fluctuating phase volume effects, and, in combustion, particle enhanced acoustical coupling should affect the dissipation and transport of turbulence energy. Our investigations, summarized here and discussed in current publications, $39,40,41,42$ isolate the purely inertial influences of the dispersed phase on reducing turbulence.

Earlier investigations on inviscid shocked flows ${ }^{43}$ and laminar viscous Eluws 44 indicate that dispersed particles significantly influence heat, mass, and momentum transport as well as wave propagation processes in the carrier gas. Generally, a dispersal of finely divided particles acts to reduce

\footnotetext{
* Senior Scientist, Chemistry and Materiale Soience Department

${ }^{+}$Graduate Student, Mechanical Engineering Department, University of California, Berkeley.

${ }^{++}$Associate Professor, Mechanical Engineering Department, University of California, Berkeley.
} 
molecular diffusion and attenuate wave propagation. We are looking for similar influences in turbulent flow. Back influences of moderately sparse particle concentrations have been theoretically investigated using a round turbulent jet configuration. 39,40 In this previous work as well as in our current work ${ }^{41,42}$ we take advantage of Crowe's formulation for gas particle forces, ${ }^{45}$ modifying the instantaneous interactions with a Monte Carlo randomization to simulate the turbulent motions.

In contrast to the present work, a substantial number of important flow situations exist where completely interactive coupling does not need to be considered. Generally, in these cases, consideration of particle dispersal alone is adequate. Particle populations are modest enough so that their effects on the flow can be ignored. Examples include the meteorological droplet dispersal predictions of Watson and Barr, ${ }^{46}$ the turbulent channel flow predictions of Peskin and $\mathrm{Kau},{ }^{47}$ and the droplet spray dispersal calculations of Dukowicz, ${ }^{48}$ who predicts the dispersed phase influences on the mean but not on the fluctuating components of the flow.

In our computational method, the coupling forces between the particles and gas are accounted for using a self-consistent, control volume formulation $^{45}$ describing forces produced by fluctuations of the gas phase and the resulting particle accelerations. The particle equations of motion are re-written in a Langrange center-of-mass system. Particle motions are treated incrementally in time, $\Delta t$. The random fluctuational gas/particle encounters are generated in an intermediate computational step. $40,41,42$ Forces considered on the particle include drag, virtual mass, Basset (boundary layer displacement), Magnus (spin), and Saffman Lift. Following determination ot the particle concentrations and trajectories, void fraction weighting is applied to the particle gas mixture equations and a new set of mixture mass modified Navier-Stokes solutions are obtained for the updated mixture field.

For illustration, our previous results ${ }^{40}$ for a round turbulent jet configuration are shown. The lateral profiles of turbulent Reynolds' stress are shown in Fig. 11 at 20 diameters from the entrance. The influence of the particles is seen to reduce significantly the Reynolds' stress relative to that obtained in a particle free gas. 
The particle concentration normalized probability distributions are shown in Figs. 12 and 13 for this round jet flow configuration. The distribution traces a transverse sweep of the computed test section. These profiles were taken at a modeled axial location, 1/2 diameter downstream from the jet entrance into the test chamber. Figure 12 shows the transverse distribution for $40 \mu \mathrm{m}$ particles while Fig. 13 shows the much greater penetration (greater particle dispersal) for $0.4 \mathrm{\mu m}$ particles. The sizes refer to the assumed common diameter of the dispersed spherical particles. Initial particle velocity distribution at the jet origin was assumed to be $10 \%$ of the mean gas phase velocity at the origin and is considered uniform in $y$, for both cases shown.

The axial auto-correlation coefficient with and without 0.18 mass loading of $40 \mathrm{~mm}$ spherical particles is shown in Fig. 14. The other flow conditions are given on the figure. A small, but consistent decrease in correlation and diminished turbulence integral scale, hence diminished eddy size and fine scale structural features, are predicted when the particles are present.

Our experiments on turbulence particle interactions are conducted at a University of California combustion wind tunnel facility, configured for Schlieren and LDV diagnostics. ${ }^{49}$. Current flow configurations include low speed, steady, mean flow in a rectangular test section. Turbulent eddy structure develops at a rearward facing step and at the trailing edge of a splitter-plate which divides the test section into equally sized upper and lower channels. The former flow configuration is shown in Fig. 15(a), while the latter is shown in Fig. $15(\mathrm{~b})$.

To measure particle influences on large structure features, for example the eddy shedding, we trace the eddy history and ensemble average the observations for a large number of eddies. The results are digitized from cinematographic observations using fast framing (5000 s. ${ }^{-1}$ ) motion picture exposures of Schlieren or (for combustion flows) shadowgraph eddy field images. Figure 16 illustrates these features with histograms. Adding larger particles produces a distinctly bimodal distribution of shedding frequency indicating an increased population of smaller (less energetic) high frequency eddies. The tendency for breakrown of the large eddy structures incrcases with particle 
mass loading. Splitter plate generated free shear turbulence power spectra LDA measurements at a mid-plane, mid-channel position just downstream of the splitter plate are shown in Fig. 17 for the three indicated Reynolds numbers, based on the channel half-depth. Our current experimental plans include measurement of the modulation of fine scale turbulent features influenced by particles using dual LDV (for particles) and LDA (for gas) diagnostics. 
THE EQUATION OF STATE OF MOLECULAR HYDROGEN AND DEUTERIUM FROM SHOCK-WAVE EXPERIMENTS TO $760 \mathrm{kbar}$

W. J. Nellis, M. Ross, A. C. Mitchell, M. van Thiel and D. A. Young

- We have carried out single shock experiments with liquid
deuterium to $200 \mathrm{kbar}(\mathrm{T} \sim 4500 \mathrm{~K})$ and reflected experi-
ments to $760 \mathrm{kbar}(\mathrm{T} \sim 7000 \mathrm{~K})$ as well as single shock
experiments with liquid hydrogen to $100 \mathrm{kbar}(\mathrm{T} \sim 3300 \mathrm{~K})$.
These results enable us to determine a spherically symmetric,
effective intermolecular pair potential that predicts iso-
therms in excellent agreement with the recent static measure-
ment to $370 \mathrm{kbar}$ of van straaten, et al. at $5 \mathrm{~K}$, but in dis-
agreement with those of shimizu, et al. to $200 \mathrm{kbar}$ at $300 \mathrm{~K}$.

The high pressure equation of state of molecular hydrogen and its transition to the metallic state is currently a subject of intense interest. In this paper we report accurate, new shock-wave results on the liquid up to a pressure of $760 \mathrm{kbar}$ and a calculated temperature of about $7100 \mathrm{~K}$. The high final temperatures and pressures achieved in the shock process allow us to determine an effective pair-potential for molecular hydrogen valid at small intermolecular separations. We have used this potential to calculate low temperature solid isotherms and to compare them with recent static measurements.

For these experiments shock waves were generated by accelerating a planar projectile to a velocity in the range $3.9-6.7 \mathrm{~km} / \mathrm{s}$ using a two-stage light-gas gun $^{50}$ and impacting the projectile onto a target containing 1 iquid $\mathrm{H}_{2}$ or $\mathrm{D}_{2}$. The expcrimcnt is based on the Raıkine-Hugoniot relations which relate measured kinematic parameters to thermodynamic variables. The diagnostic system was described previously. 51 Liquid $\mathrm{D}_{2}$ specimens were also double-shocked by reflecting a shockwave off an aluminum (alloy 1100) anvil to achieve pressures four times higher than could be achieved by a single shock. The technique has been described previously. 52 For the double-shock $D_{2}$ experiments the mixing circuit used to multiplex two detector signals onto a single oscilloscope was modified somewhat because of the short shock transit times being measured ( $110 \mathrm{~ns}$ ). The cryogenic targets have also been described. 53 The coolant was 1 iquid $\mathrm{H}_{2}$ for all experiments. The cryogenic design was modified because the heat of vaporization per unit volume is significantly less for 1 iguid $\mathrm{H}_{2}$ 
than for coolants used previously, like liquid $\mathrm{N}_{2}$ or Ar. The initial state was close to the saturation curve at $20.2 \mathrm{~K}$. Au-Fe vs chromel thermocouples were calibrated in place. Sample temperature was used to obtain initial sample density. 54,55 The Hugoniot equation of state for the metal impactors and walls or base plates were published earlier, 56 as was the calculation of the release isentrope of the aluminum base plate. ${ }^{53}$ Small corrections were made for thermal contraction as previously noted. ${ }^{53}$. The error analysis has also been published. 51,56 Changes in experimental details from our other experiments will be published elsewhere.

The data are plotted in Figs. 18 and 19 and are compared with earlier work of van Thiel, et al. ${ }^{57,58,59}$ and Dick and Kerley. ${ }^{60}$. Figure 18 shows the principal Hugoniot up to $U .21$ Mbar and the reflected $v_{2}$ Hugonlot data near 0.76 Mbar. These higher pressure points were reflected from the lower point at 0.21 mbar. Fig. 19 shows the $\mathrm{H}_{2}$ principal Hugoniot points. The present shockwave experiments have the highest accuracy of any to date on $\mathrm{H}_{2}$ and. $\mathrm{D}_{2} \cdot$

One goal of our work has been to determine a spherically symmetric, effective intermolecular potential which can be used with accurate theoretical models to calculate the equation of state of condensed hydrogen. In 1978 Silvera and Goldman ${ }^{61}$ (SG) carried out a very careful theoretical study of the deuterium isotherm at $4 \overrightarrow{\mathrm{K}}$ and up to $26 \mathrm{kbar}$ using selt-consistent photon theory and determined an intermolecular potential that best fit these data. ${ }^{62}$ Subsequently we showed that their potential would also predict the fluid isotherms of $\mathrm{H}_{2}$ and $\mathrm{D}_{2}$ from 75 to $300 \mathrm{~K}$ up to $20 \mathrm{kbar}$ and the melting curves to 57 kbar in agreement with experimental data. ${ }^{62}$ In these calculations we used soft-sphere perturbation theory for the fluid properties and lattice dynamics for the solid with anharmonic corrections. The melting curves were computed by equating the Gibbs free energies and pressures of the two phases. However, this limited data base is insufficient to determine the short range intermolecular repulsions that dominate the high temprature-high pressure shocked states, and as a result the SG potential predicts Hugoniot curves that are too steep. We found that if this potential were modified for $\mathrm{r}<2.55 \AA$ it was possible to compute the theoretical Hugoniots shown in Figs. 18 and 19, $34 d$ still maintain the excellent fit to the lower pressure fluid, solid and melting data. 
Our new potential is

$$
\begin{array}{cc}
\phi=\phi_{S G} & r>r_{C} \\
\phi=A \exp \left[B\left(r-r_{c}\right)-C\left(r-r_{c}\right)^{2}-D\left(r-r_{c}\right)^{3}-E\left(r-r_{c}\right)^{3}\left(r-r_{1}\right)\right] \\
r<r_{C}
\end{array}
$$

where $A=3.98823 \times 10^{-14} \mathrm{erg}, \mathrm{B}=4.76940 \AA^{-1}, \mathrm{C}=2.25457 \AA^{-2}, \mathrm{D}=$ $0.955189 \AA^{-3}, E=0.248158 \AA^{-4}, r_{1}=1.2 \AA$, and $r_{C}=2.55 \AA$.

The calculation of the Hugoniot curve and the statistical mechanical theory used to compute the thermodynamic properties of compressed fluid hydrogen are described in previous publications. $62,63,64$ In these calculations the vibrational and rotational properties are assumed to be unaffected by compression and were calculated as in the gas phase. The actual change in the vibrational frequency under compression to pressure up to $600 \mathrm{kbar}$ at $300 \mathrm{~K}$ reported by Sharma, et al. ${ }^{65}$ is too small be observed along the Hugoniot. Molecular dissociation is expected to be negligible at these densities. The theoretical Hugoniot curves were calculated with fluid perturbation theory and Monte Carlo calculations. Perturbation theory is particularly useful in a study of this type in which a best fit pair potential is sought and fast calculational methods are desirable. To check the accuracy of the perturbation theory we carried out Monte Carlo calculations and determined that the predicted Hugoniots were generally softer than perturbation theory by about 18 in volume, but well within the \pm 38 experimental error. The theoretical curves shown in Figs. 18 and 19 are based on Monte Carlo results. Also indicated on the figures are some of the calculated temperatures. The high temperatures achieved in these experiments provide information about the pair potential down to molecular separations of about $1.6 \AA$, or comparable to separations at which the molecular-metallic transition is belleved to take place.

Pressure versus volume measurements, made using diamond anvils, have been reported by Shimizu, et al. ${ }^{66}$ up to 200 kbars at 300 Kelvins, and by van straaten, et al. ${ }^{67}$ up to 370 kbars at 5 Kelvins. These two sets of results, shown in Fig. 20, are in serious disagreement. The disagreement is somewhat 
worse than it appears since the $300 \mathrm{~K}$ isotherm is below the $5 \mathrm{~K}$ curve. Also shown is a $5 \mathrm{~K}$ isotherm calculated using lattice dynamics and the intermolecular potential that best fitted our shock-wave data. The theoretical curve is in very good agreement with the results of van straaten, et al. It is worth emphasizing that the shock-wave method has nothing in common with diamond anvil experiments and represents a totally independent determination of the $\mathrm{H}_{2}$ equation of state.

We have recalculated the molecular to monatomic metal transition using a molecular equation of state based on our new intermolecular potential and metal equations of state calculated by the APW method ${ }^{68}$ and third order perturbation theory. ${ }^{69}$ zero point properties and electron correlation energy were included. These theories predict metallic transitions at 3.1 Mbar (APW) and 3.6 Mbar (third order). The new results do not differ substantially from earlier work. ${ }^{70}$ This result assumes metallization will occur by molecular dissociation. However, experimental work on iodine ${ }^{71}$ has demonstrated that a molecular crystal can become conducting, without dissociation, as a result of the crossing of the filled valence band of the molecule with its empty conduction band. Some preliminary theoretical calculations for a solid at $0 \mathrm{~K}$ indicate that hydrogen may undergo the same transition. 72,73 
NUCLEAR-EXPLOSIVE-DRIVEN EQUATION-OF-STATE EXPERIMENT

W. J. Nellis, A. C. Mitchell, R: Olness, M. van Thiel

- We are in the process of fielding a shock-impedance-match experiment $d r i v e n$ by a nuclear explosive. This equationof-state experiment is a joint effort between $H$ Division, A Division (W. P. Crowley, D. Hardin), B Division (J. White, G. Repp), and L Division (R. Heinle, H. Finn, T. Valk). The experiment is similar to that described by C. Ragan. $74 \mathrm{~A}$ planar shockwave will be generated in $\mathrm{Pb}$, transmitted into an Al baseplate, and shock-impedance-matched into samples which include $\mathrm{Be}, \mathrm{Cu}$, and $\mathrm{Pb}$. Expected pressures are about 10 Mbar in $\mathrm{Al}$ and $\mathrm{Be}$ and about $20 \mathrm{Mbar}$ in $\mathrm{Cu}$ and $\mathrm{Pb}$. Measured shock transit times will be about $500 \mathrm{~ns}$. The detectors will be electrical shorting pins developed in 1975.75 


\section{REFERENCES}

1. A. K. McMahan, M. T. Yin and M. L. Cohen, Phys. Rev. B, in press.

2. J. A. Moriarty, Phys. Rev. B 10, 3075 (1974); 16, 2537 (1977).

3. A. D. Pasternak, Mater. Sci. Eng. 3, 65 (1968).

4. D. Glötzel and A. K. McMahan, Phys. Rev. B 20, 3210 (1979). A. K. McMahan, H. L. Shriver and B. Johansson, Phys. Rev. B 23, 5016 (1981).

5. R. Grover, R. N. Keeler, F. J. Rogers, J. Phys. Chem. Solids 30, 2091 (1969). R. Grover, J. Phys. Chem. Solids 32, 2539 (1971). R. Grover, I. C. Getting, G. C. Kennedy, Phys. Rev. B 7, 567 (1972).

6. LASL Shock Hugoniot Data, compiled S. P. Marsh (U. Cal. Press, Berkeley, 1979). Compendium of Shock Wave Data, UCRL-50108, Vol. 1-3, 1977, Gen. Ed. M. van Thiel.

7. I. N. Makarenko, A. M. Nikolaenko and S. M. Stishov, Phys. Letters 49A, 257 (1974).

8. J. Ramakrishnan and G. C. Kennedy, J. Phys. Chem. Solids 41, 301 (1980).

9. D. B. Boercker, F. J. Rogers and H. E. Dewitt, The Electron Collision Frequency in Plasmas, Lawrence Livermore National Laboratory, Livermore, CA, UCRL-86598, 1981 (to appear in Phys. Rev. A 25).

10. R. H. Williams and H. E. Dewitt, Phys. Fluids 12, 2326 (1969).

il. F. J. Rogers, H. E. DeWitt, and D. B. Boercker, phys. Lett. $82 \mathrm{~A}, 331$ (1981).

12. See for instance, W. Gautschi and W. F. Cahill, in Handbook ot Mathematical Functions, edited by M. Abramowitz and I. A. Stegun (Dover, New York, 1965).

13. R. Radtke and K. Gunther, J. Phys. D 9, 1131 (1976); K. Gunther, M. M. Popovic, S. S. Popóvic, and R. Radtke, J. Phys. D 9, 1139 (1976).

14. L. Spitzer, Jr., Physics of Fully Ionized Gases (Interscience, New York, 1956).

15. J.-P. Hansen and I. R. McDonald, Phys. Kev. A 23, 2041 (1981); M. Baus, J.-P. Hanisen and L. Sjogru, Pliys. Lett. B2A, In! (1.9B1); $\tau_{1}$, Gjogren, J.-P. Hansen, and E. L. Pollock, Phys. Rev. A 24, 1544 (1981).

16. F. J. Rogers, D. B. Boercker, H. E. Dewitt, in H-Division Quarterly Report, Lawrence Livermore National Laboratory, Livermore, CA, UCID-18574-81-4 (1981). 
17. A. I. Larkin, Sov. Phys. JETP 11,1363 (1960).

18. A. A. Barker, J. Chem. Phys. 55, 1751 (1971).

19. F. J. Rogers, Phys. Rev. A23, 1008 (1981).

20. C. Deutsch and M. M. Gambert, J. Math. Phys. 17, 1077 (1976).

21. J. P. Hansen and I. P. McDonald, Phys. Rev. Lett. $\underline{41}, 1379$ (1978).

22 H. E. DeWitt, H. C. Graboske, M. S. Cooper, Astrophysical J. 181, 439 (1973).

23. S. G. Brush, H. Sahlin, E. Teller, J. Chem. Phys. $45,2101(1966)$.

24. A. Alastuey and B. Jancovici, Astrophysical J. 226, 1034 (1978).

25. N. Itoh, H. Totsuji, S. Ichimaru, H. E. DeWitt, Astrophysical J. 234, 1079 (1979).

26. W. L. Slattery, G. D. Doolen, H. E. DeWitt, Phys. Rev. A21, 2087 (1980).

27. H. E. DeWitt, UCID-18754-81-2.

28. J. P. Hansen, G. M. Torrie, P. Vieillefosse, Phys. Rev. Al6, 2153 (1977).

29. H. DeWitt and F. Rogers, UCID - 18574-80-1.

30. Y. Rosenfeld, private communication, preprint.

31. H. Totsuji, Phys. Rev. A24, 1077 (1981).

32. W. T. Ashurst (1974), Ph.D. Dissertation, University of California at Davis-Livermore.

33. W. G. Hoover, D. J. Evans, R. B. Hickman, A. J. C. Ladd, W. T. Ashurst, and B. Moran, Phys. Rev. A 22, 1690 (1980). See also C. H. Fawcett, Hobbies 63,41 (1958).

34. H. C. Anderson, J. Chem. Phys. 72, 2384 (1980).

35. For similar three-dimensional work see D. M. Heyes, J. J. Kim, C. J. Montrose, and T. A. Litovitz, J. Chem. Phys. 73,3987 (1980).

36. A. J. C. Ladd and W. G. Hoover, "Microscopic Theory of Plasticity", to be submitted to Phys. Rev. B (1982).

37. W. G. Hoover, N. E. Hoover, and w. C. Moss, J. Appl. Phys. 59, 829 (1979).

38. Hill, R., The Mathematical Theory of Plasticity, p. 50-52, Oxford Press, 1960.

39. A. C. Buckingham, "Dusty Gas Influences on Transport in Turbulent Erosive Propellant Flow", AIAA Journal 19, (4), 501-508 (1981). 
40. A. C. Buckingham and W. J. Siekhaus, "Simulating the Interaction Between Turbulence and Particles in Erosive Flow and Transport" in Numerical Methods in Laminar and Turbulent Flow, eds. C. Taylor and B. A. Schrefler, Pineridge Press, Swansea, U.K., 929-942 (1981).

41. Buckingham, A. C., W. J. Siekhaus, J. O..Keller, J. Ellzey, G. Hubbard, J. W. Daily, "Computed and Experimental Interactions Between Eddy Structure and Dispersed Particles in Developing Free Shear Layers," Lawrence Livermore National Laboratory UCRL-86936 (November, 1981), submitted to AIAA/ASME Joint Fluid, Plasma, Thermodynamics and Heat Transfer Conference (St. Louis, Mo. June 7-11, 1982).

42. Buckingham, A. C., W. J. Siekhaus, J. O. Keller, J. Ellzey, G. Hubbard, J. W. Daily, "Computations and Experiments on Interactions Between Inert Particles and Turbulence in Developing Free Shear", Lawrence Livermore National Laboratory UCRL-87963 (January, 1982), submiteed to N1nth U.S. National Congress of Applied Mechanics (NATCAM), (Cornell University, Itaca, N.Y. June 21-25, 1982).

43. Carrier, G. F., J. Fluid Mech. 4 , 376 (1955).

44. Marble, F. E., "Dynamics of Dusty Gases," Ann. Review of Fluid Mech. $\underline{2}$, 397 (1970).

45. Crowe, C. T., "Vapor-Droplet Flow Equations," Univ. of California, Lawrence Livermore National Laboratory UCRL-51877 (August 18, 1975).

46. Watson, C. W., and S. Barr, "Monterárlo Simulation of Turbulent Transport of Airborne Contaminants," Los Alamos National Laboratory, Univ. of California, LA-6103 (January 1976).

47. Peskin, R. L. and C. J. Kau, "Numerical Simulation of Particle Motion in Turbulent Gas-Solid Channel Flows," J. Fluid Engr. 101, 319 (Sept., 1979).

48. Dukowicz, J. K., "A Particle-Fluid Numerical Model for Liquid Sprays," J. of Comp. phys. $35(2), 229$ (1980).

49. Pitz, R. W. and J. W. Daily, "Experimental Study of Combustion in a Turbulent Free Shear Layer Formed at a Rearward Facing Step, "AIAA Paper \#81-0106 in AIAA 19th Aerospace Sciences Meeting (St. Louis, Mo., Jan. $12-15,1981)$.

50. A. H. Jones, W. M. Isbell, and C. J. Maiden, J. Appl. Phys. 37, 3493 (1966).

51. A. C. Mitchell and W. J. Nellis, Rev. Sci. Instrum. 52, 347 (1981).

52. W. J. Nellis, F.H. Ree, M. van Thiel and A. C. Mitchell, J. Chem. Phys. 75, 3055 (1981).

- W. J. Nellis and A. C. Mitchell, J. Chem. Phys. 73, 6137 (1980).

54. R. Prydz, "The Thermodynamic Properties of Deuterium", National Bureau of Standards, Report 9276, 1967. 
55. H. M. Roder, L. A. Weber, R. D. Goodwin, "Thermodynamic and Related Properties of Parahydrogen from the Triple Point to $100^{\circ} \mathrm{K}$ at Pressures to 340 Atmospheres", National Bureau of Standards, Monograph 94, 1965.

56. A. C. Mitchell and W. J. Nellis, J. Appl. Phys. 52, 3363 (1981).

57. M. van Thiel and B. J. Alder, Mol. Phys. 10, (1966).

58. M. van Thiel, M. Ross, B. L. Hord, A. C. Mitchell, W. H. Gust, M. J. D'Addario and R. N. Keeler, Phys. Rev. Lett 31, 979 (1973).

59. M. van Thiel, L. B. Hord, W. H. Gust, A. C. Mitchell, M. D'Addario, K. Boutwell, E. Wilburger and B. Barrett, Phys. Earth Planetary Inter. 9, 57, (1974).

60. R. D. Dick and G. I. Kerley, J. Chem. Phys. $\underline{73}, 5264$ (1980).

61. I. F. Silvera and V. V. Goldman, J. Chem. Phys. 69, 4209 (1978).

62. M. Ross and D. A. Young, Phys. Lett. 78A, 4631 (1980); D. A. Young and M. Ross, J. Chem Phys. 74, 6950 (1981).

63. M. Ross, J. Chem. Phys. 73,4445 (1980).

64. M. Ross and F. H. Ree, J. Chem. Phys. $\underline{73}, 6146$ (1980).

65. S. K. Sharma, H. K. Mao and P. M. Bell, Phys. Rev. Bl3, 5154 (1976).

66. H. Shimizu, E. M. Brody, H. K. Mao and P. M. Bell, Phys. Rev. Lett 47 , 128 (1981).

67. J. van Straaten, J. Rinke Wijngarden and I. F. Silvera, Phys. Rev. Lett., $\underline{48}, 97(1981) \ldots$

68. M. Ross and A. K. McMahan, Phys. Rev. B13, 5154 (1976).

69. J. Hammerberg and N. W. Ashcroft, Phys. Rev. B9, 409 (1974).

70. M. Ross, J. Chem. Phys. 60,3634 (1974).

71. K. Takemura, S. Minomura, O. Shimomura and Y. Fujii, Phys. Rev. Lett. $\underline{45}$, 1881 (1980).

72. C. Friedli and N. W. Ashcroft, Phys. Rev. Bl6, (1977).

73. S. Chakravarty, J. H. Rose, D. Wood and N. W. Ashcroft, Phys. Rev. B24, 1624 (1981).

74. Charles E. Ragan III, Phys. Rev. A $\underline{21}, 458$ (1980).

75. A. C. Mitchell, in $\mathrm{H}$ Division Quarterly Report, Lawrence Livermore National Laboratory, Livermore, CA, UCRL-50028-75-2 (1975). 


\section{Figure Captions}

Fig. I Relative total energies of bcc, hcp and fcc structures vs. volume, as calculated by the GPT method. a) $\mathrm{Na}$, b) $\mathrm{Mg}$.

Fig. 2 Relative total energies of the bcc, hcp and fcc structure of Al vs. volume, as calculated by (a) the LMTO method, and (b) the GPT method.

Fig. 3 The bcc-fcc total energy difference for Al vs. volume, as calculated by the GPT method and by the LMTO method with and without d states.

Fig. 4 Calculated zero temperature bulk moduli as function of relative volumes. Values of $\mathrm{B}$ and $\mathrm{dln} B / \mathrm{dV}$ derived from static and ultrasonic experimental data range over values shown by $\Phi$ and the shaded cones along right axis. Static compression data for Na up to compressions of 308 ranges over values indicated by $I$.

Fig. 5 Calculated and experimental values of the Grineisen coeffirient as function of relative volume.

Fig. 6 Electron-proton effective potentials for $\mathrm{kT}=1 \mathrm{eV}, \rho=0.0166$ $\mathrm{g} / \mathrm{cm}^{3}, \alpha_{e p}=80 \mathrm{a}_{0}$. The Barker pseudopotential (Eq. (2)), the Deutsch pseudopotential (Eq. (4)), and the Planck-Larkin pseudopotential (Eg. (3)) are shown.

Fig. 7 The same as Fig. 6 except $\mathrm{kT}=2 \mathrm{eV}$.

Fig. 8 Hypernetted chain electron-proton distribution functions for the Deutsch and Planck-Larkin pseudopotentials, corresponding to Fig. 7.

Fig. 9 OCP Helmholtz free energy.

Fig. 10 Plastic dilatation increases the pressure.

Fig. 11 Calculated radial prufiles uf Lurbulent Reynoldo ctrese between ruandjel cenleiline and wall at indivabed partigle monnentrations.

Fig. 12 Calculated normalized $40 \mu \mathrm{m}$ radial particle concentration distributions between round turbulent jet centerline and wall at indicated times.

Fig. 1.3 Calculated normalized 0.4 um radial particle concentration distributions between round turbulent jet centerline and wall at indicated times.

Fig. 14 Calculated turbulent axial (streamwige) vcloolty cumponent auto-correlation coefficient with and without particles.

Fig. 15 (a) Experimental two-dimensional blow-down turbulent two-phase flow combustion chamber with rearward facing step turbulence generator.

(b) Experimental two-dimensional blow-down turbulent two-phase flow combustion chamber with dividing flow splitter plate turbulence generator. 
Fig. 16 Histograms of turbulent eddy shedding features from Schlieren fast framing movie records, rearward facing step configuration.

Fig. 17 Turbulent power spectra LDA measurements in splitter plate generated mixing layer at three test flow speeds and corresponding Reynolds numbers.

Fig. 18 Principal and reflected deuterium Hugoniots.

Fig. 19 Principal liquid hydrogen Hugoniot

Fig. 20 Solid hydrogen isotherms.

$\mathrm{mpd} / \mathrm{ks} / 0081 \mathrm{Q}$ 


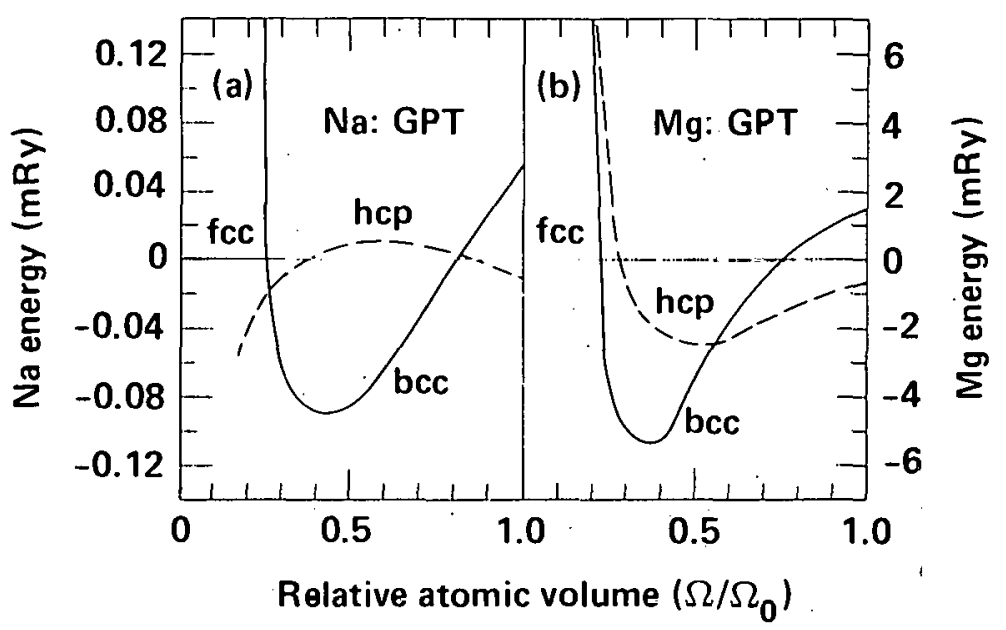

Fig. 1 


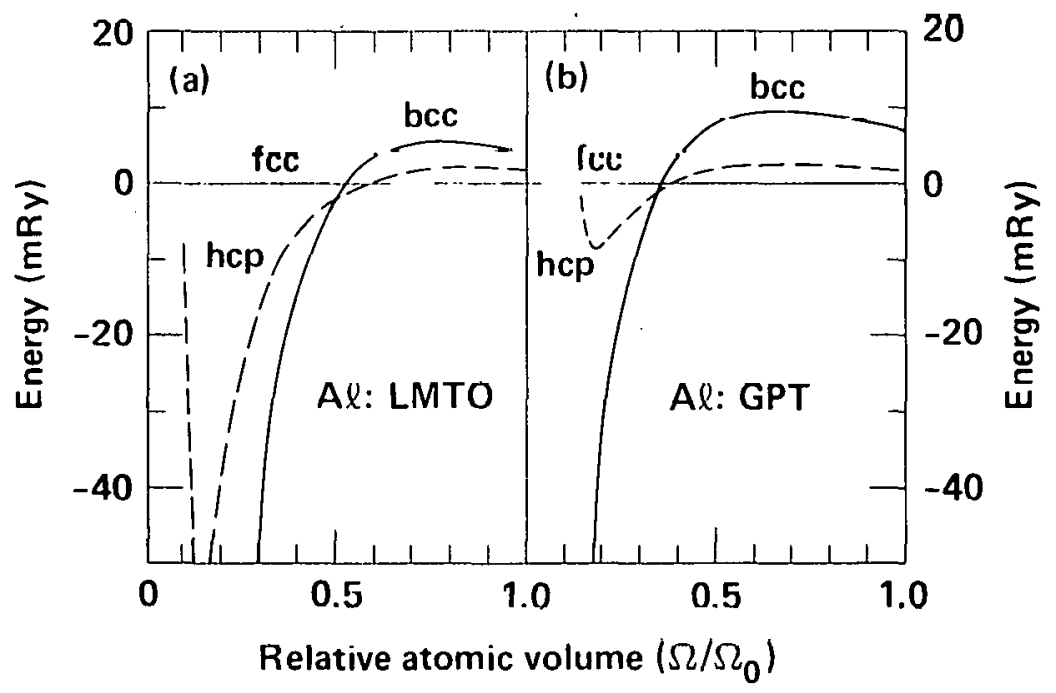

Fig. 2 


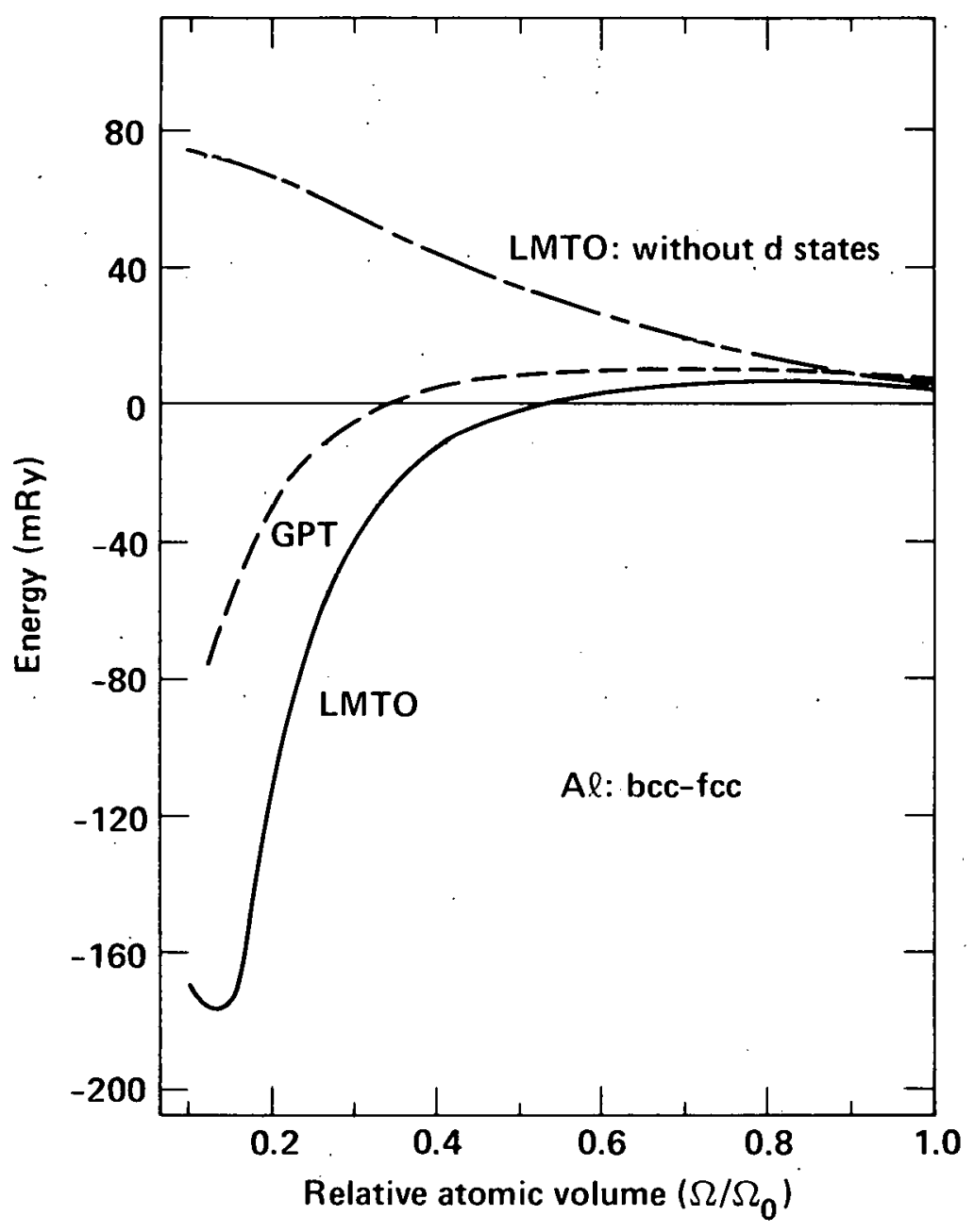

Fig. 3 


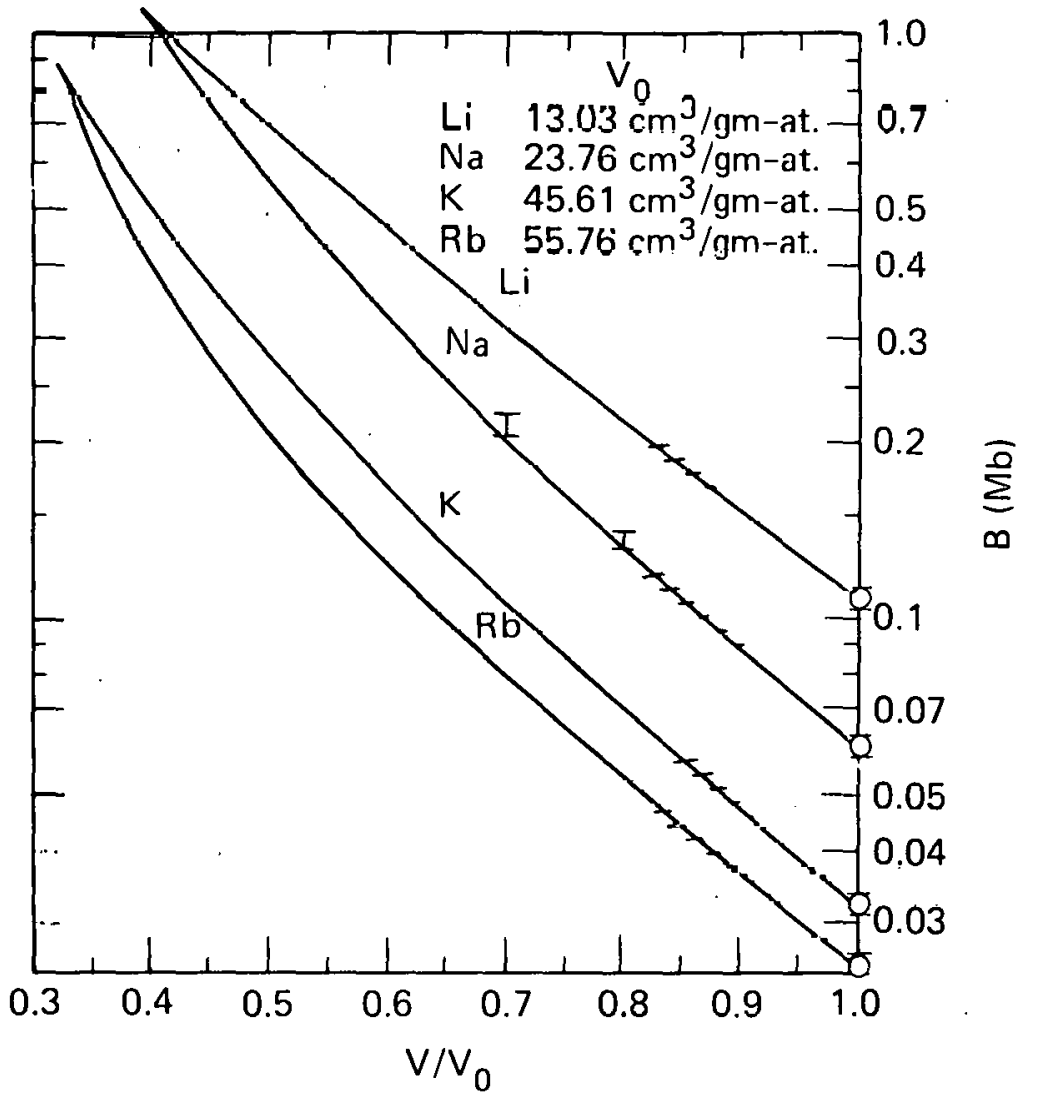

Fig. 4 


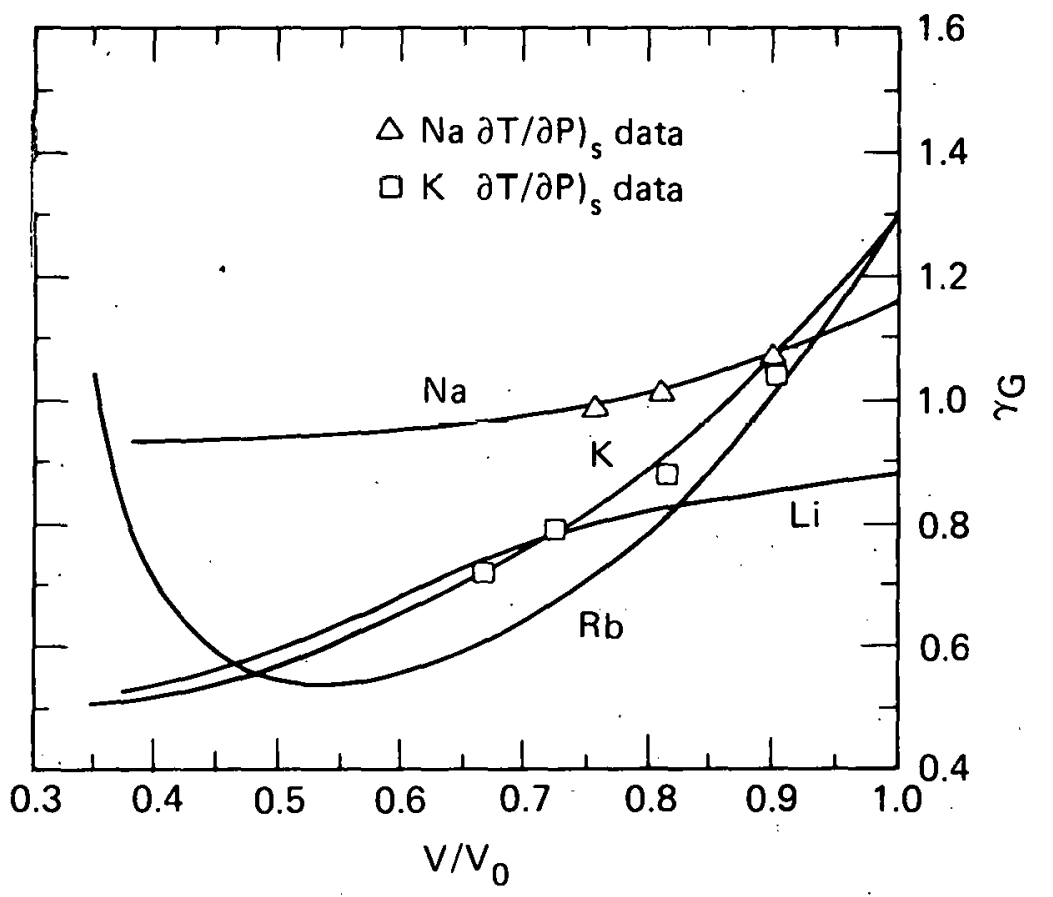

Fig. 5 


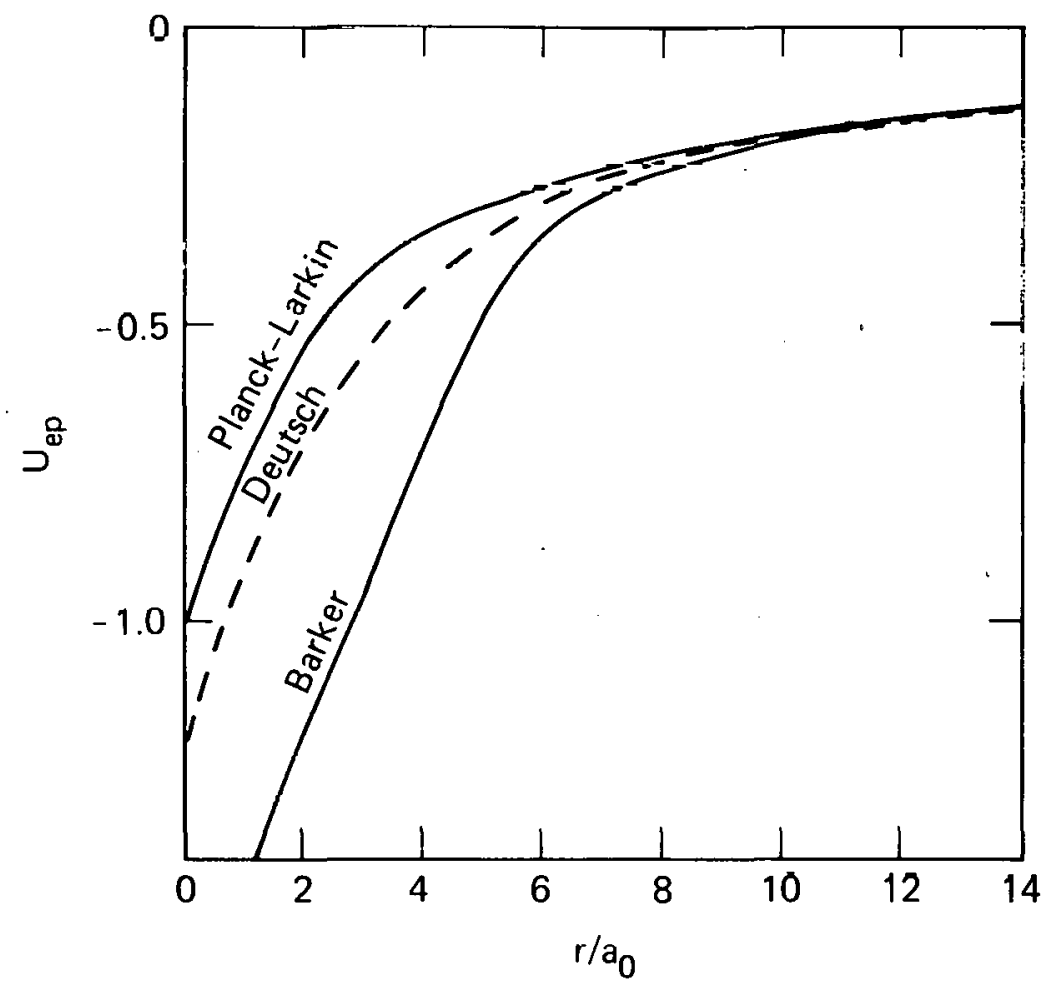

Fig. 6 


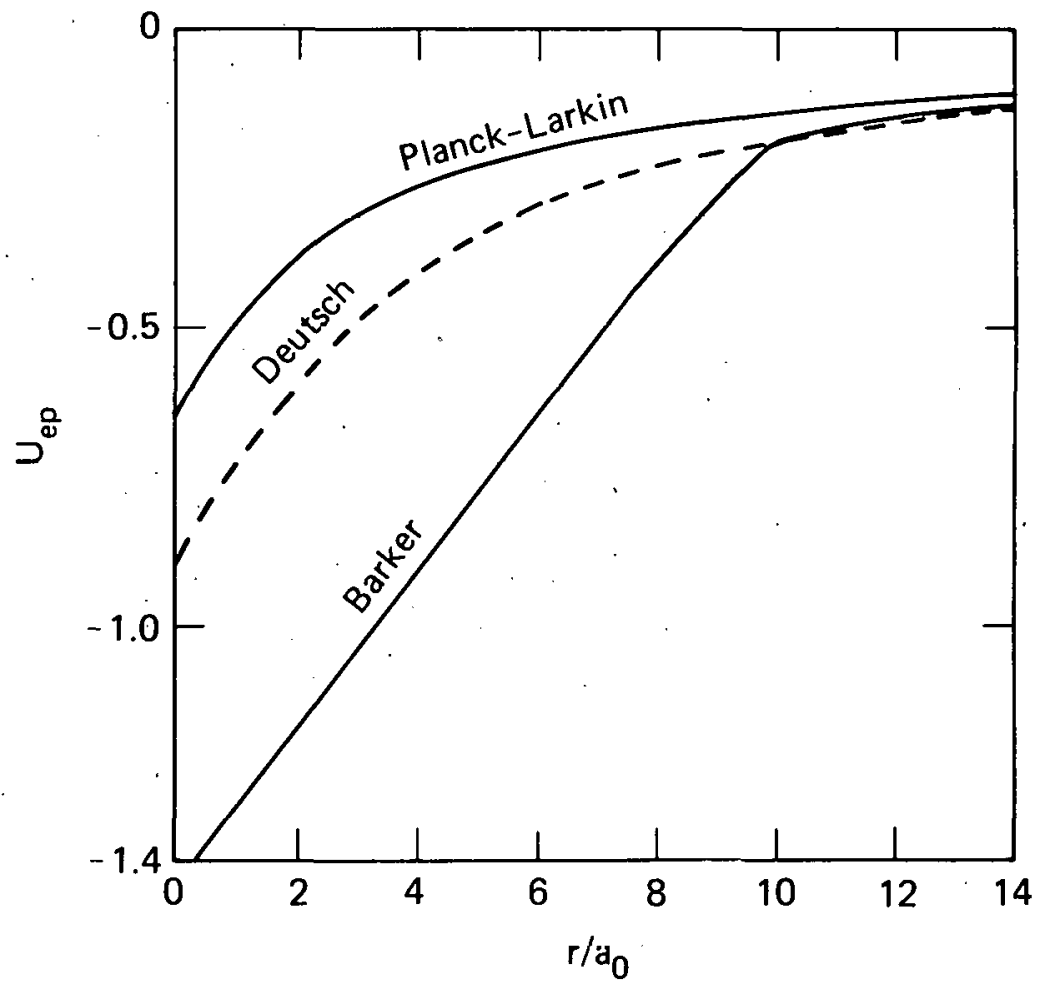

Fig. 7 


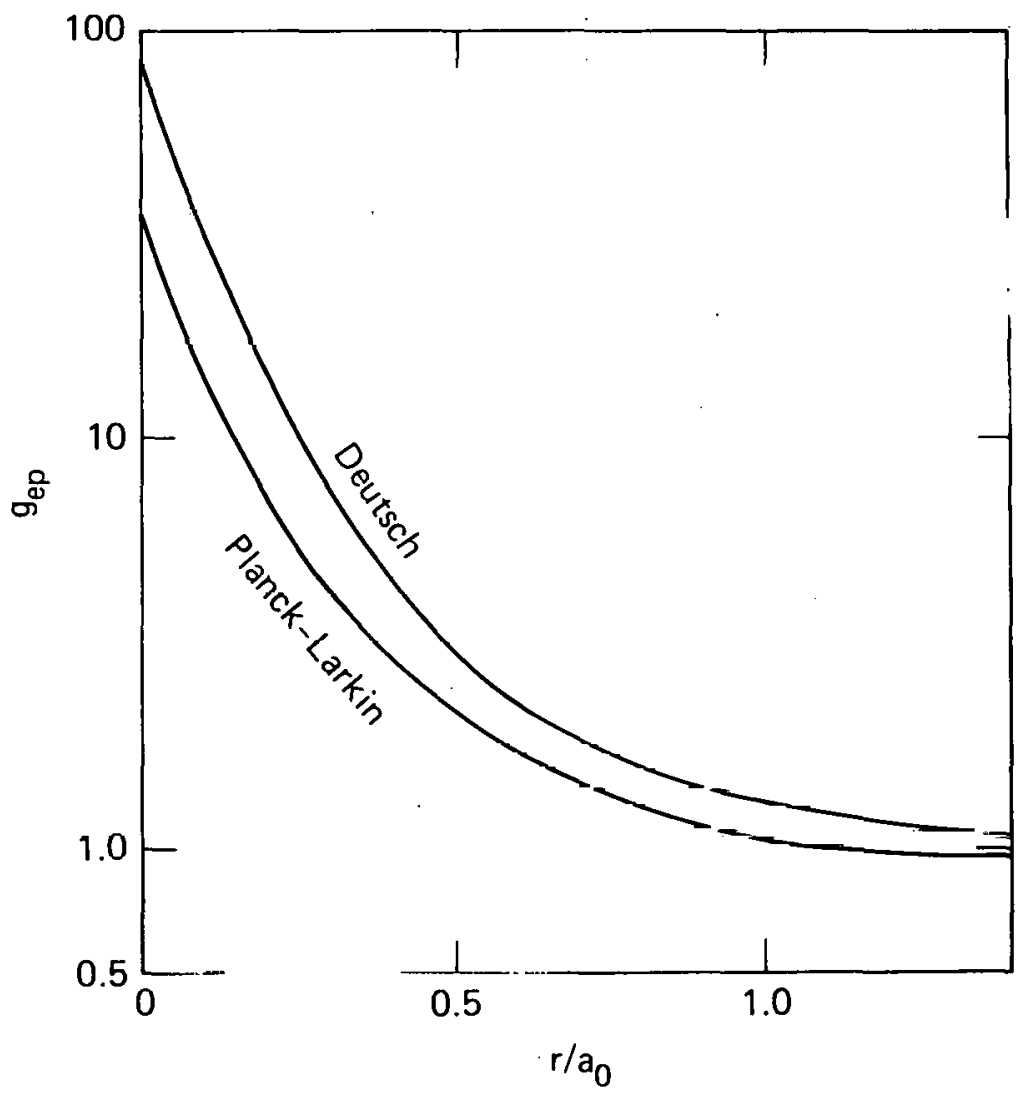

Fig. 8 


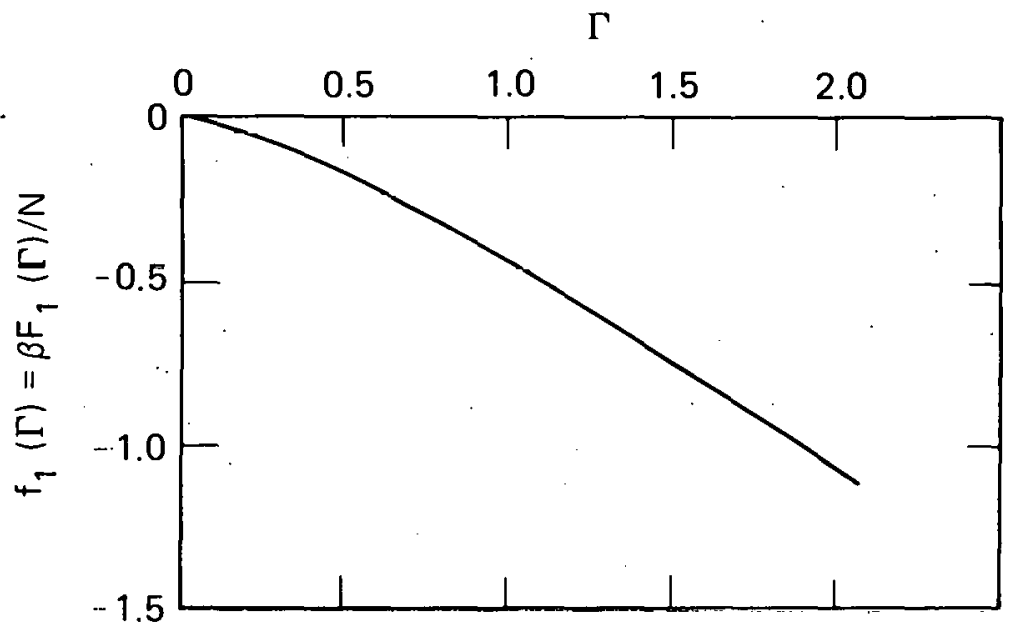

Fig. 9 


$$
\text { 政 }
$$




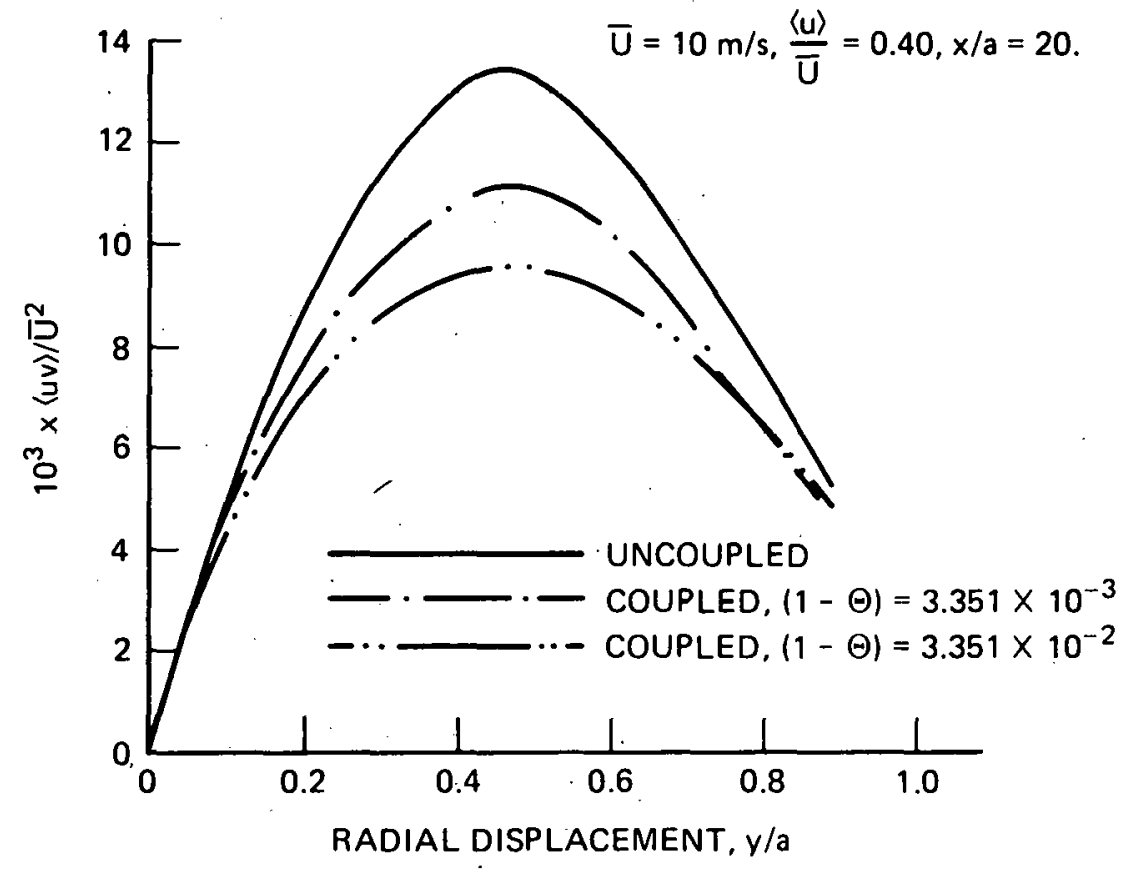

Fig. 11 


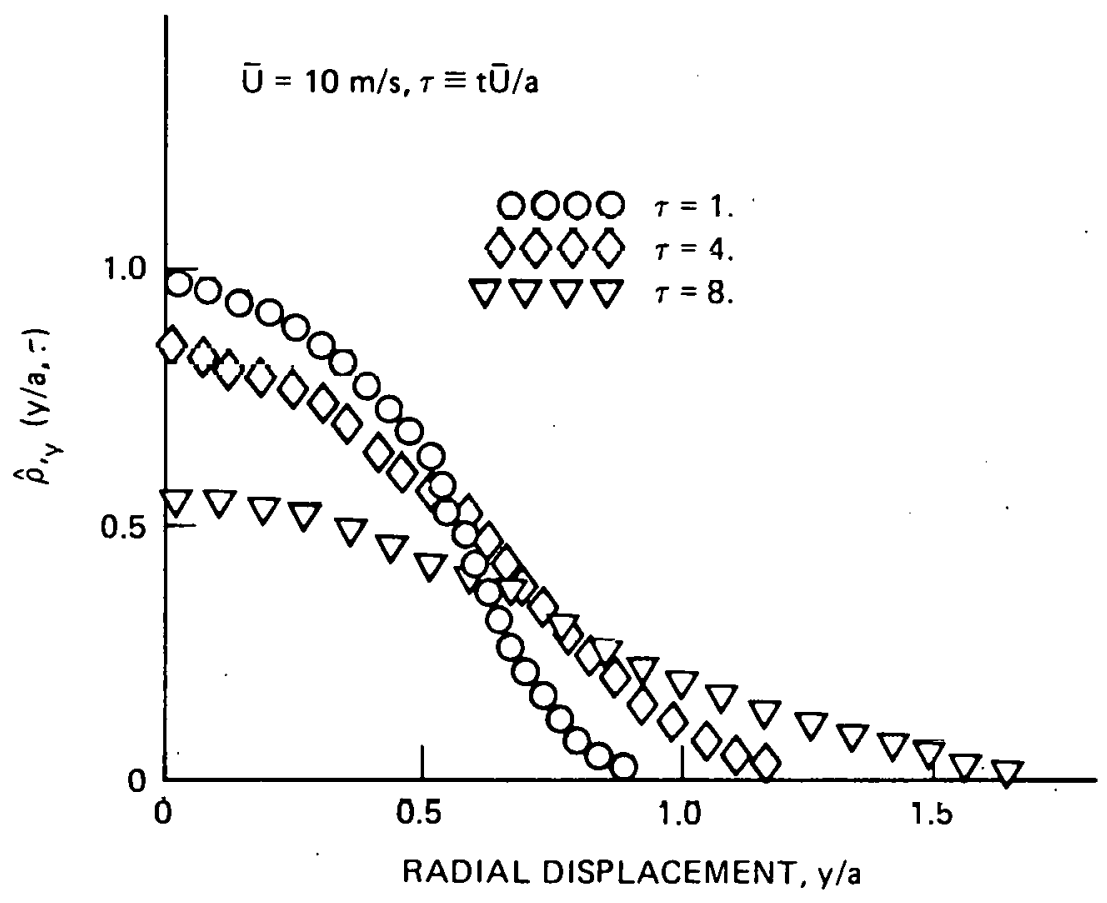

Fig. 12 


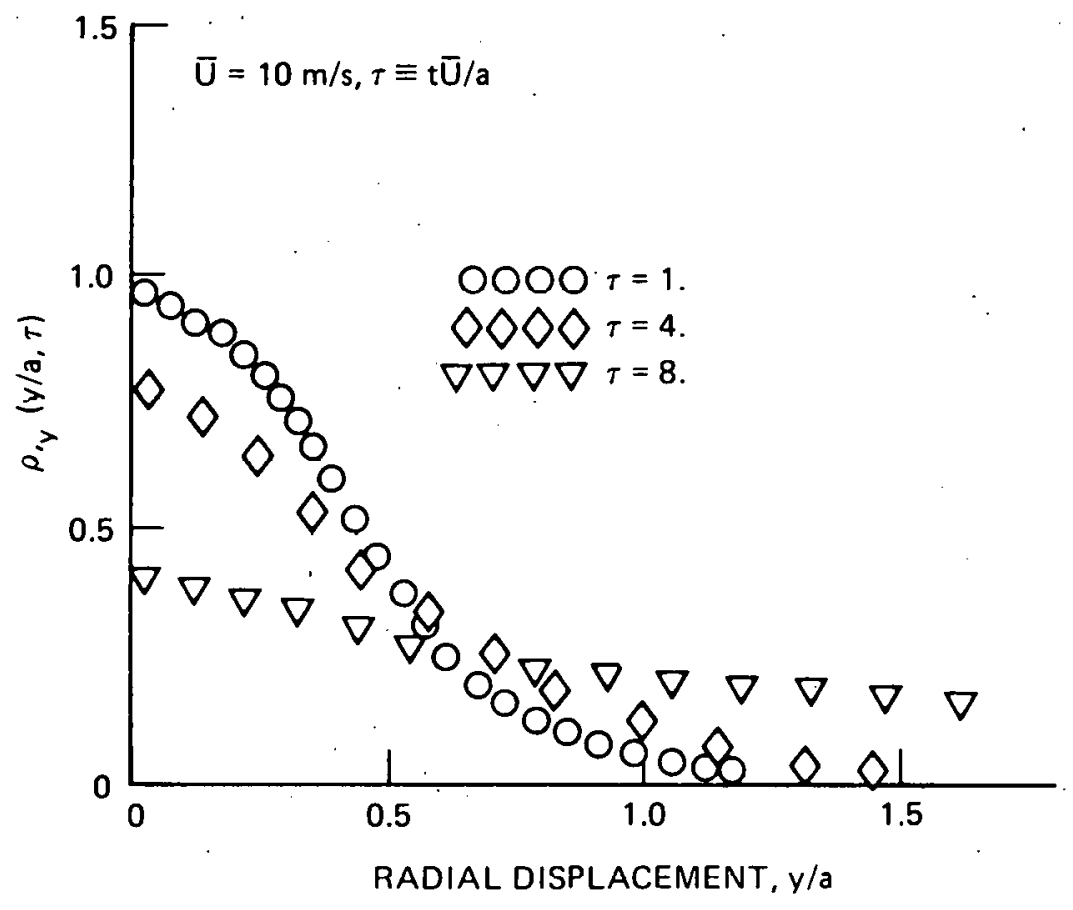

Fig. 13 


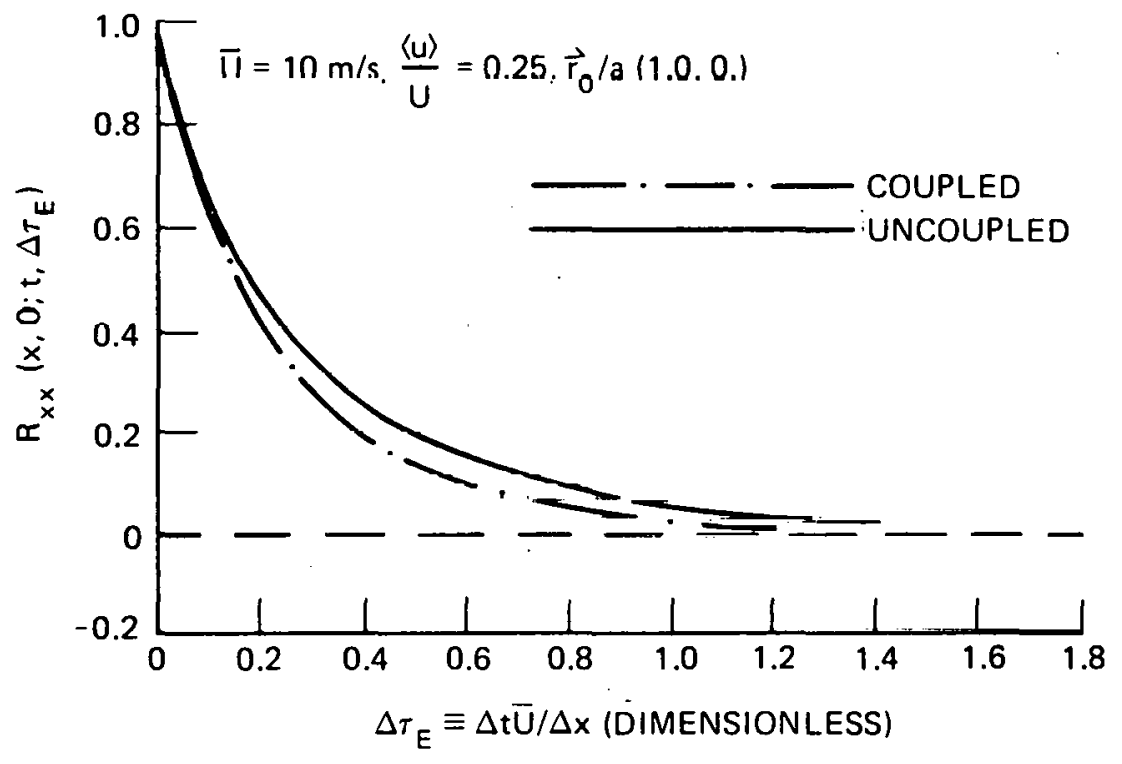

Fig. 14 


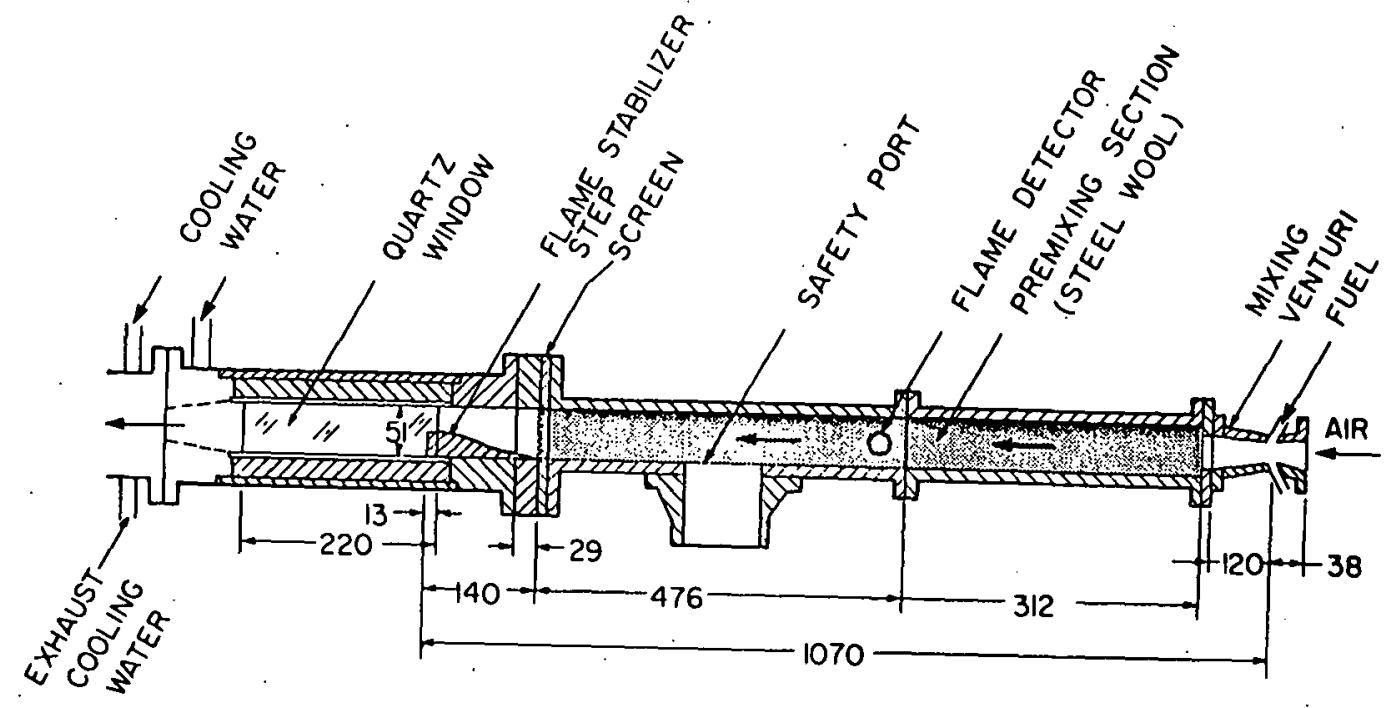

Fig. 15 (a)

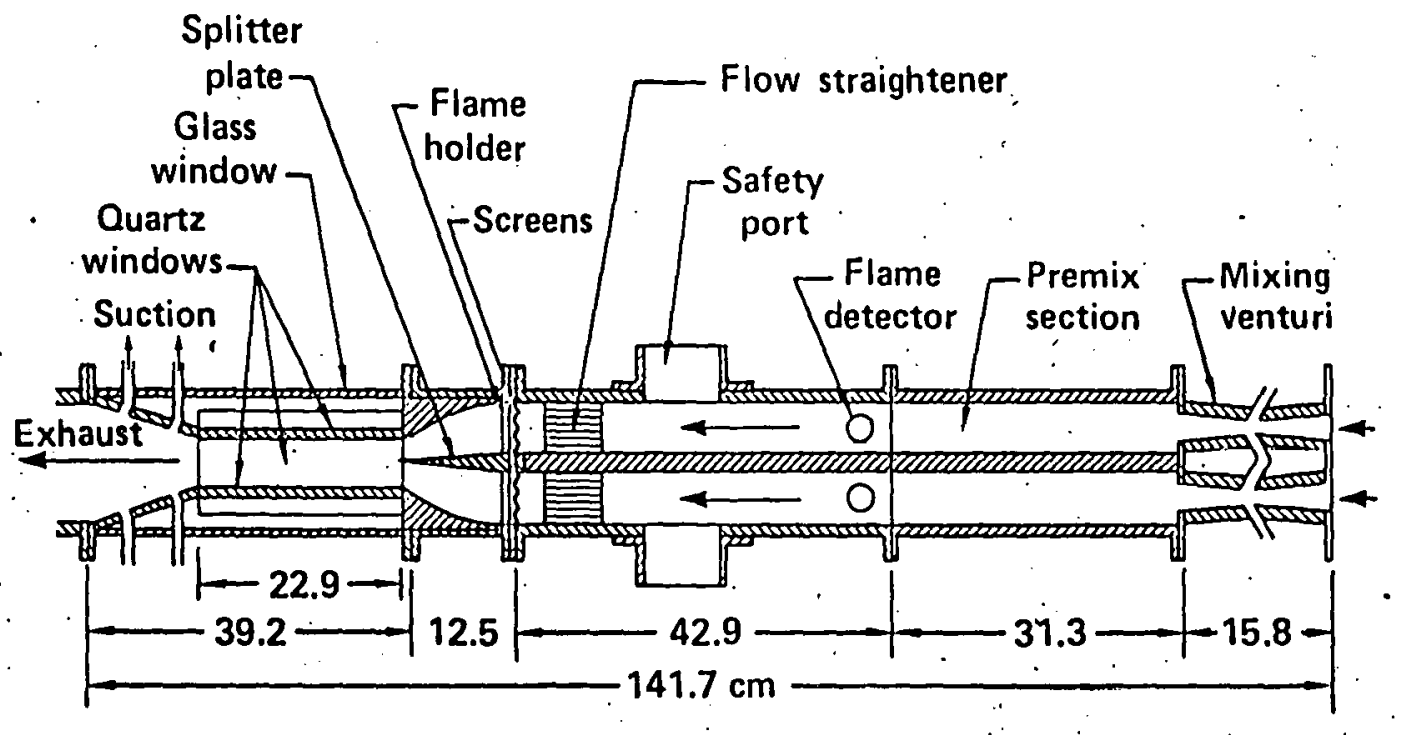

Fig. 15 (b) 
$\operatorname{Re}\left(\mathrm{cm}^{-1}\right)=9092, U_{\infty}=13.28 \mathrm{~m} / \mathrm{s}, T=298 \mathrm{~K}$

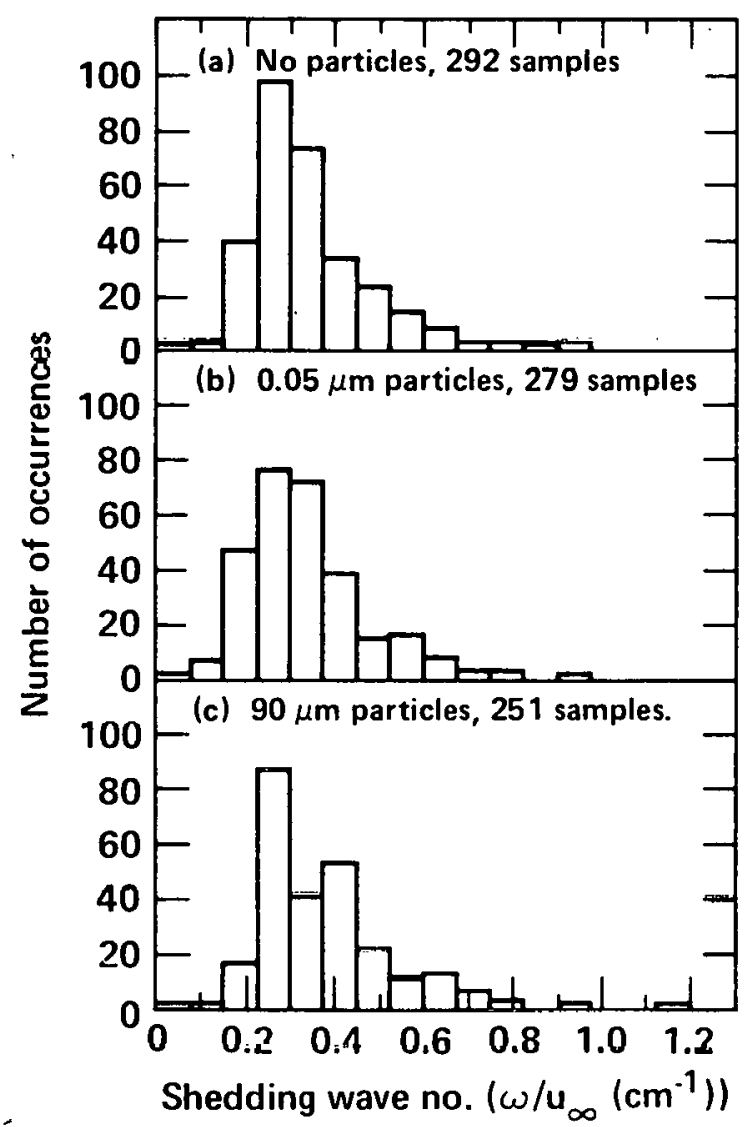

Fig. 16 


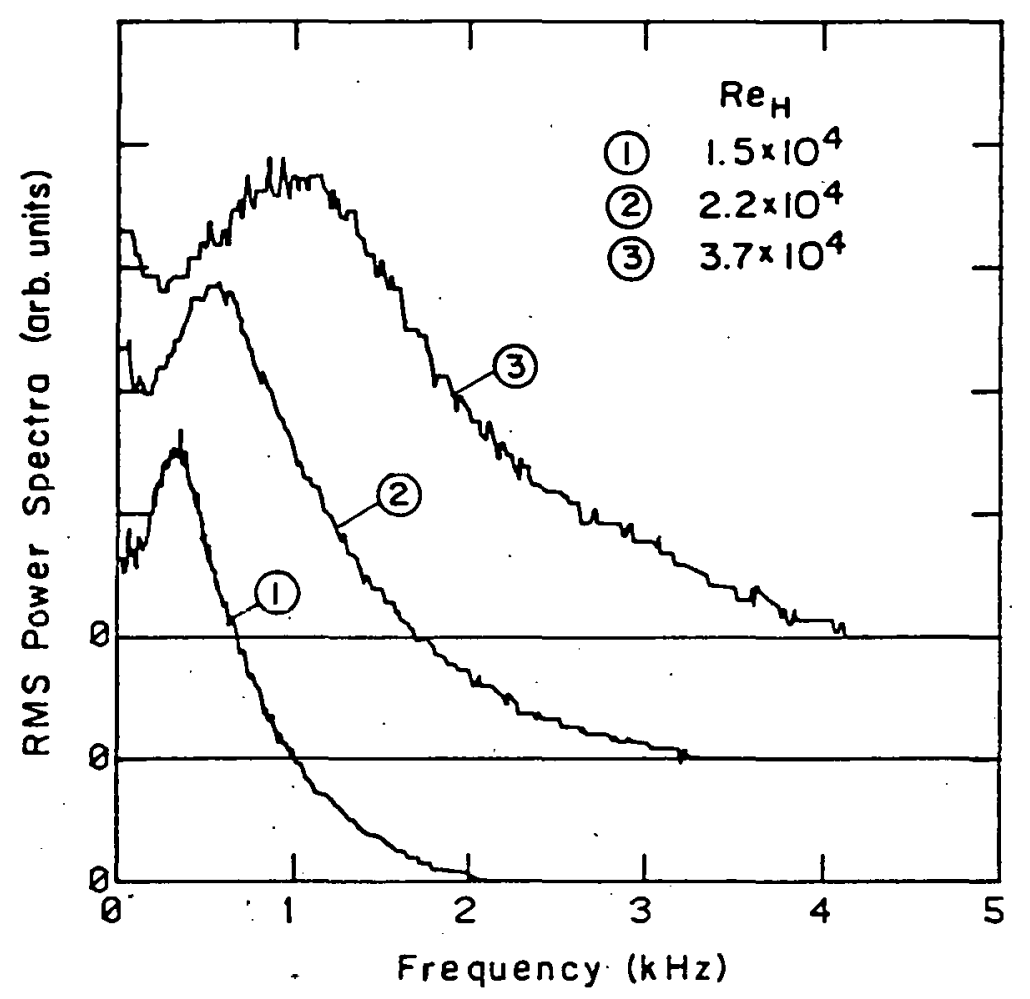

Fig. 17 


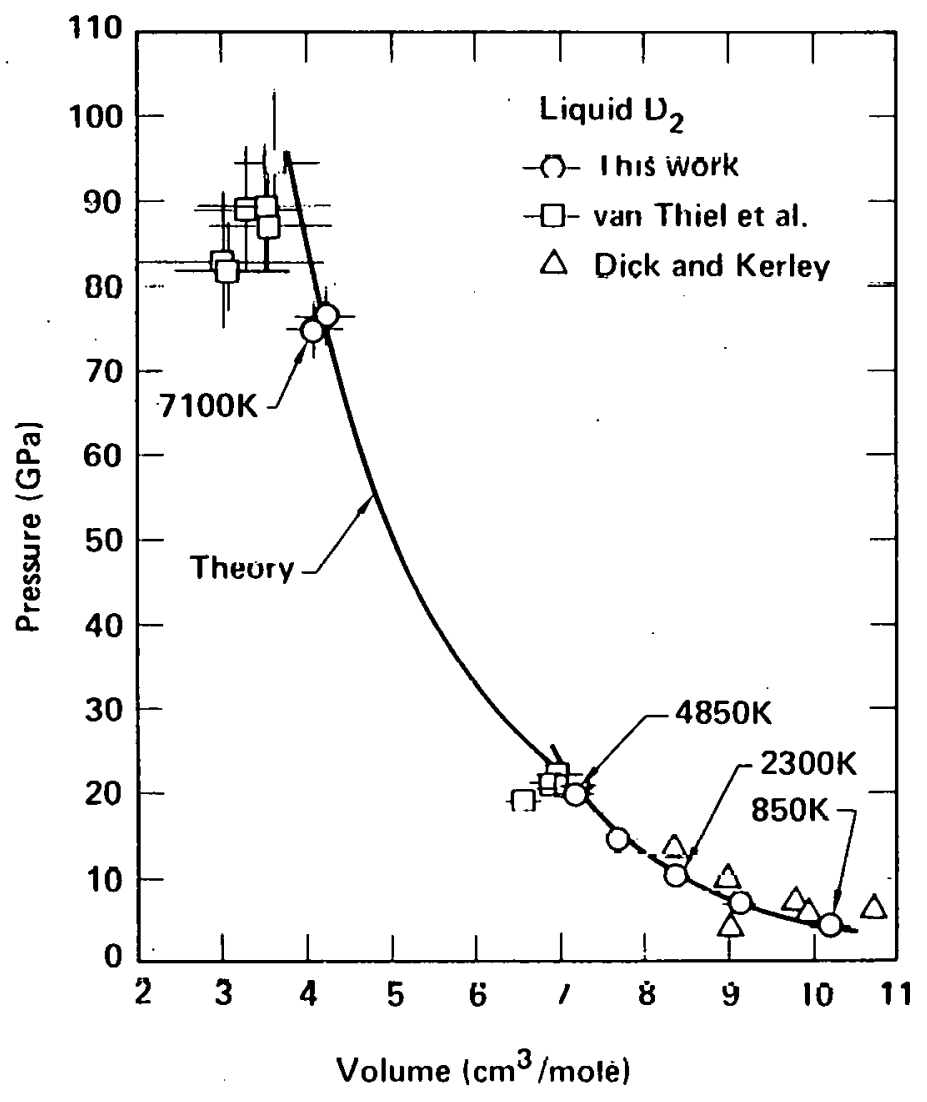

Fig. 18 


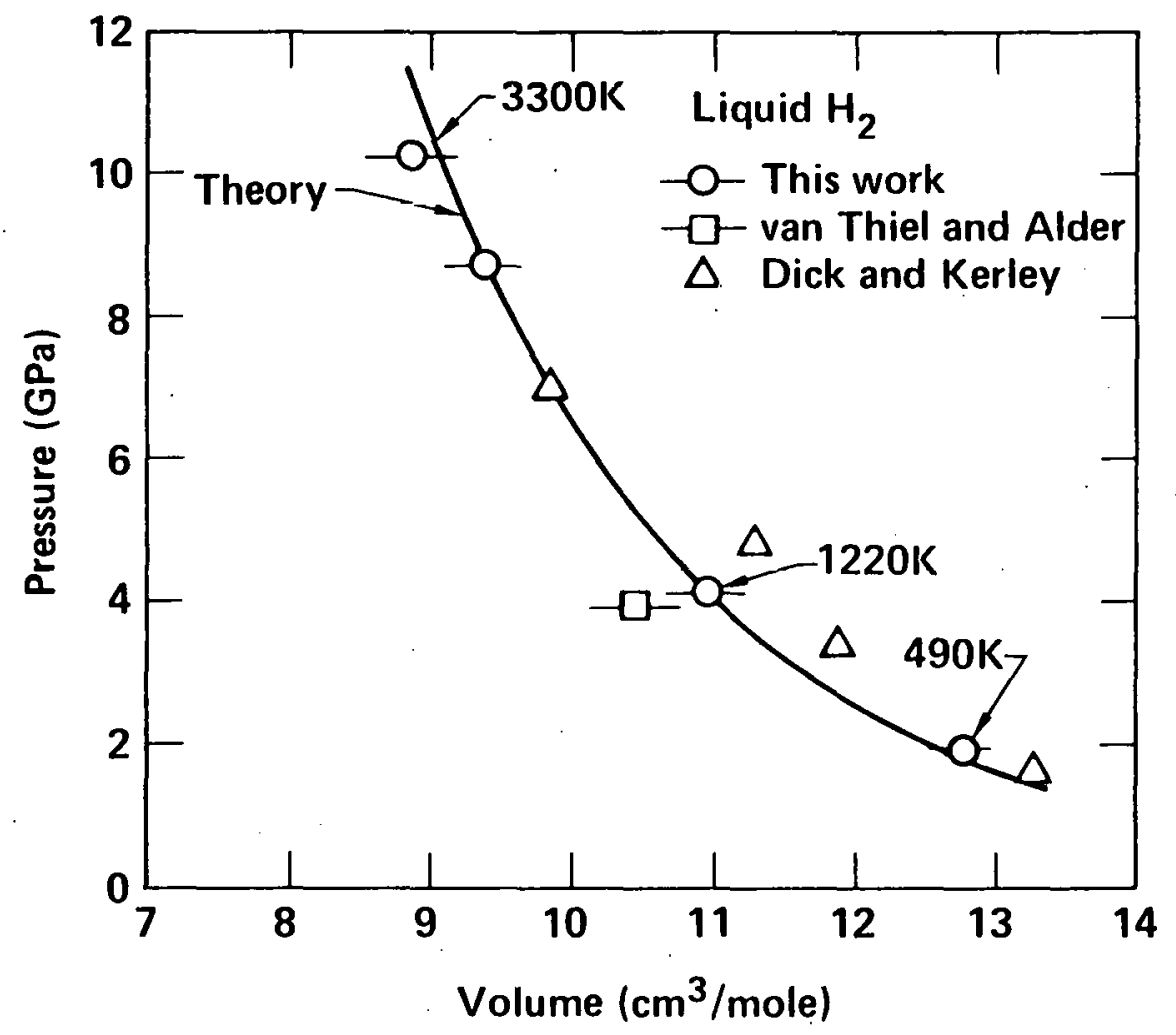

Fig. 19 


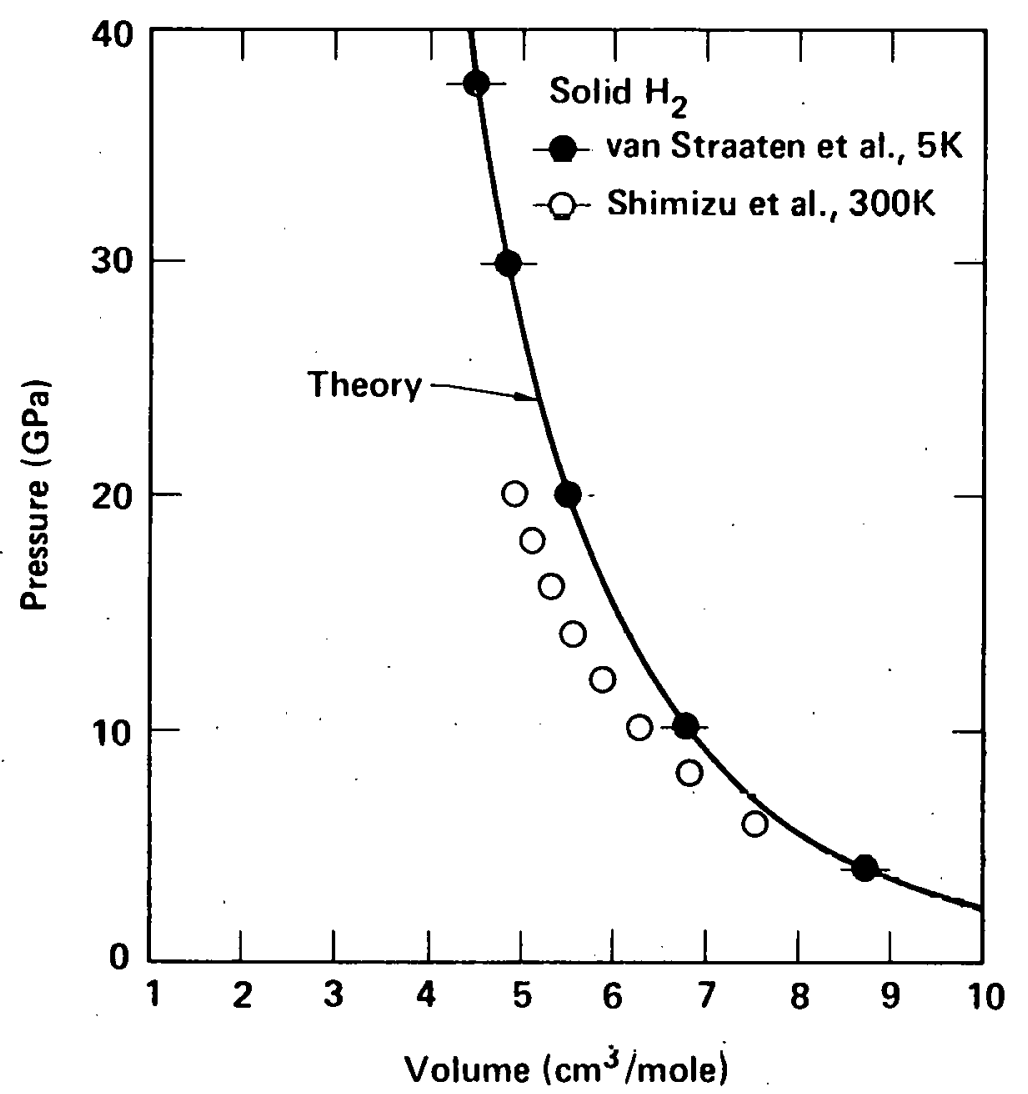

Fig. 20 


\section{DISC LAIMER}

This document was prepared as an account of work sponsored by an agency of the United States Government. Neither the Inited States Government nor the I niversity of California nor any of their employees, makes any warranty, express or implied, or assumes any legal liability or responsibility for the accuracy, completeness, or usefulness of any information, apparatus, product, or process disclosed, or represents that its use would not infringe privately owned rights. Reference herein to any specific commercial products, process, or service by trade name, trademark, manufacturer, or otherwise, does not necessarily constitute or imply its endorsement, recommendation, or favoring by the I nited States Government or the University of California. The views and opinions of authors expressed herein do not necessarily state or reflect those of the United States Government thereof, and shall not be used for advertising or product endorsement purposes.

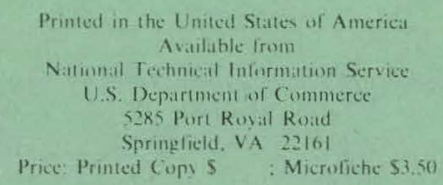

\begin{tabular}{|c|c|c|c|}
\hline Page Range & $\begin{array}{l}\text { Domestic } \\
\text { Price }\end{array}$ & Page Range & $\begin{array}{l}\text { Domestic } \\
\text { Price }\end{array}$ \\
\hline $001-025$ & $\$ 5.00$ & $326-350$ & $\$ 18.00$ \\
\hline $026-050$ & 6.00 & $351-375$ & 19.00 \\
\hline $051-075$ & 7.00 & $376-400$ & 20.00 \\
\hline $076-100$ & 8.00 & $401-425$ & 21.00 \\
\hline $101-125$ & 9.00 & $426-450$ & 22.00 \\
\hline $126-150$ & 10.00 & $451-475$ & 23.00 \\
\hline $151-175$ & 11.00 & $476-500$ & 24.00 \\
\hline $176-200$ & 12.00 & $501-525$ & 25.00 \\
\hline $201-225$ & 13.00 & $526-550$ & 26.00 \\
\hline $226-250$ & 14.00 & $551-525$ & 27.00 \\
\hline $251-275$ & 15.00 & $526-550$ & 28.00 \\
\hline $276-300$ & 16.00 & 601 -up 1 & \\
\hline $301-325$ & 17.00 & & \\
\hline
\end{tabular}

${ }^{1}$ Add 2.00 for each additional 25 page increment from 601 pages up. 


$$
1
$$

